\title{
Downregulation of TREM2 Expression Exacerbates Neuroinflammatory Responses Through TLR4- Mediated MAPK Signaling Pathway in a Transgenic Mouse Model of Alzheimer's Disease
}

\author{
John Bosco Ruganzu \\ Xi'an Jiaotong University \\ Xiaoqian Peng \\ Xi'an Jiaotong University \\ Yingying $\mathrm{He}$ \\ Xi'an Jiaotong University \\ Xiangyuan Wu \\ Xi'an Jiaotong University \\ Quzhao Zheng \\ Xi'an Jiaotong University \\ Bo Ding \\ Xi'an Jiaotong University \\ Chengheng Lin \\ Xi'an Jiaotong University \\ Weina Yang ( $\nabla$ wn_yang@mail.xjtu.edu.cn ) \\ Xi'an Jiaotong University https://orcid.org/0000-0002-4874-1188
}

\section{Research Article}

Keywords: Alzheimer disease, Microglia, $\beta$-amyloid, Triggering receptor expressed on myeloid cells 2, Neuroinflammation

Posted Date: March 2nd, 2021

DOI: https://doi.org/10.21203/rs.3.rs-251906/v1

License: (1) This work is licensed under a Creative Commons Attribution 4.0 International License. Read Full License 
Version of Record: A version of this preprint was published at Molecular Immunology on February 1st, 2022. See the published version at https://doi.org/10.1016/j.molimm.2021.12.018. 


\section{Abstract}

Activation of glial cells and neuroinflammation play an important role in the onset and development of Alzheimer's disease (AD). Triggering receptor expressed on myeloid cells 2 (TREM2) is a microgliaspecific receptor in the brain that is involved in regulating neuroinflammation. However, the precise effects of TREM2 on neuroinflammatory responses and its underlying molecular mechanisms in AD have not been studied in detail. Here, we employed a lentiviral-mediated strategy to downregulation of TREM2 expression on microglia in the brain of APPswe/PS1dE9 (APP/PS1) transgenic mice and BV2 cells. Our results showed that TREM2 downregulation significantly aggravated AD-related neuropathology including $A \beta$ accumulation, peri-plaque microgliosis and astrocytosis, as well as neuronal and synapseassociated proteins loss, which was accompanied by a decline in cognitive ability. The further mechanistic study revealed that downregulation of TREM2 expression initiated neuroinflammatory responses through toll-like receptor 4 (TLR4)-mediated mitogen-activated protein kinase (MAPK) signaling pathway and subsequent stimulating the production of pro-inflammatory cytokines in vivo and in vitro. Moreover, blockade of p38, JNK, and ERK1/2 inhibited the release of tumor necrosis factor-a (TNF-a), interleukin-1 $\beta$ (IL-1 $\beta$ ), and interleukin-6 (IL-6) induced by A $\beta_{1-42}$ in TREM2-knocked down BV2 cells. Taken together, these findings indicated that TREM2 might be a potential therapeutic target for AD and other neuroinflammation related diseases.

\section{Introduction}

Alzheimer's disease (AD) is the most common age-dependent neurodegenerative disorder characterized by severe memory loss, unusual behavior, and a progressive decline in cognitive function. The classical neuropathological hallmarks of $A D$ include extracellular $\beta$-amyloid ( $A B$ )-associated plaques, intraneuronal tau-associated neurofibrillary tangles (NFTs), and loss of neurons and synapses. Increasing evidence demonstrated that neuroinflammation plays a significant role in the development and pathology of $A D$ $[1-3]$. The neuroinflammatory cascade is principally initiated through microglia and astrocytes. Microglia, the brain resident innate immune cells, are the critical cell type that contributes to neuroinflammatoryrelated pathology during AD [4]. Microglia activation was found to be related to the interaction between $A \beta$ with several receptors including toll-like receptor 4 (TLR4) in AD patients and animal models [5]. A $\beta$ binding to TLR4 activates the mitogen-activated protein kinase (MAPK) signaling pathway by its downstream molecule myeloid differential protein 88 (MyD88) and TNF-receptor-associated factor 6 (TRAF6) $[6,7]$. When activated, microglia aggregate around $A \beta$ plaques and produce various proinflammatory cytokines and inflammatory mediators, such as tumor necrosis factor-a (TNF-a), interleukin-1 $\beta$ (IL-1 $\beta$ ), interleukin-6 (IL-6), reactive oxygen species, and nitric oxide [3, 8]. Pro-inflammatory cytokines promote amyloid precursor protein (APP) production and the process of APP proteolytic cleavage to increase the production of $A \beta$, resulting in a vicious cycle in $A D$ [9-11].

Triggering receptor expressed on myeloid cells 2 (TREM2) is a type I transmembrane receptor of the immunoglobulin superfamily, which is selectively and highly expressed on microglia in the brain $[12,13]$. In AD, increased expression of TREM2 has been confirmed in patients and in mouse models of amyloid 
and tau pathology, which suggests that microglia may require TREM2 to prevent plaque expansion and protect neurons [14-17]. TREM2 interacts with its adaptor DNAX-activating protein of $12 \mathrm{kDa}$ (DAP12) to regulate critical functions of microglia including enhancement of phagocytosis rate, promotion of microglial proliferation and survival, as well as inhibition of pro-inflammatory cytokines production [12, $18,19]$. Experiments performed in vitro studies found that TREM2 is strongly involved in $A \beta_{1-40}$ and $A \beta_{1-42}$ uptake by microglia $[20,21]$. An in vivo study demonstrated that TREM2 deficiency could aggravate $A \beta$ plaques burden due to decreased phagocytic clearance of $A \beta$ in $5 x F A D$ mice [22]. However, overexpression of TREM2 in $A D$ animal models resulted in reduced $A \beta$ plaques load $[20,23,24]$. TREM2 also influences $A D$ through modulating inflammatory signaling. In microglia, the knockdown or knockout of TREM2 signaling increased TNF-a, IL-1 $\beta$, IL-6, and nitric oxide synthase-2 (NOS2) transcription, while overexpression of TREM2 decreased gene transcription of TNF-a, IL-1 $\beta$, and NOS2 $[20,25,26]$. Previous studies reported that the deficiency of TREM2 enhanced pro-inflammatory cytokines production and exacerbated neuroinflammation in P301S mice and senescence-accelerated mouse prone 8 mice $[27,28]$. Overexpressing of TREM2 in P301S mice reduced levels of pro-inflammatory cytokines [20,29]. Furthermore, TLR-mediated inflammation was negatively regulated by TREM2 in vitro studies [30-33]. Anti-inflammatory effects of TREM2 after TLR stimulation have been confirmed in several cell lines [3032]. Conversely, TLR stimulation induced a higher release of pro-inflammatory cytokines TNF-a and IL-6, by bone marrow-derived macrophages lacking TREM2 [33]. However, the precise effects of TREM2 on neuroinflammation and its underlying molecular mechanisms in APPswe/PS1dE9 (APP/PS1) transgenic mouse model of $A D$ are still unclear. Therefore, in the current study, a lentiviral-mediated strategy was employed to silence TREM2 in the brain of APP/PS1 mice and cultured BV2 cells. We tested the hypothesis that downregulation of TREM2 expression could exacerbate neuroinflammatory responses through TLR4-mediated MAPK signaling pathway.

\section{Materials And Methods}

\section{Reagents and antibodies}

Thioflavin S (Th-S), bovine serum albumin (BSA), Hexafluoro-2-propanol (HFIP), and dimethylsulfoxide (DMSO) were obtained from Sigma-Aldrich (Saint Louis, MO, USA). A $\beta_{1-42}$ was purchased from APeptide Co., Ltd (Shanghai, China). Anti-synaptophysin (Syn), anti-PSD-95, anti-total-p38, anti-phospho-p38 (Thr180/Tyr182), anti-total-JNK, anti-phospho-JNK (Thr183/Tyr185), anti-total-ERK1/2, anti-phosphoERK1/2 (Thr202/Tyr204), anti-TLR4, anti-MyD88, and FITC-conjugated goat anti-rabbit IgG were obtained from Cell Signaling Technologies (Beverly, MA, USA). Anti-TREM2, anti-ionized calcium-binding adapter molecule 1(Iba1), and anti-NeuN were purchased from Abcam (Cambridge, MA, USA). Anti- $\beta$-actin was obtained from Santa Cruz (CA, USA). Anti-TRAF6 was purchased from Proteintech (Wuhan, China). Antiglial fibrillary acidic protein (GFAP), anti-MOAB2, and Cy3-conjugated goat anti-mouse IgG were obtained from Novus Biologicals (Littleton, CO, USA). Mouse TNF- $\alpha$, IL-1 $\beta$, and IL- 6 enzyme-linked immunosorbent assay (ELISA) kits were purchased from Mei-mian Biotechnology (Yancheng, China). Mouse $A \beta_{1-40}$ and $A \beta_{1-42}$ ELISA kits were obtained from Invitrogen (Carlsbad, CA, USA). Horseradish peroxidase (HRP)- 
conjugated goat anti-mouse and goat anti-rabbit IgG were purchased from Pierce (Rockford, IL, USA). Biotinylation HRP-conjugated goat anti-rabbit IgG was obtained from Zhongshan Golden Bridge Biotechnology Corporation (Beijing, China). Nitrocellulose filter (NC) membrane was purchased from Pall Corporation (New York, NY, USA). Enhanced chemiluminescence (ECL) detection reagents were obtained from Bio-Rad Laboratories, Inc. (Hercules, CA, USA). Mouse direct polymerase chain reaction (PCR) kit was purchased from Bimake (Houston, TX, USA). RNAiso Plus, Prime Script ${ }^{\text {TM }}$ RT Master Mix, Premix $\mathrm{Taq}^{\mathrm{TM}}$, and SYBR® Premix Ex Taq ${ }^{\mathrm{TM}}$ II were obtained from Takara Biotechnology Corporation (Dalian, China). Fetal bovine serum (FBS) was purchased from Biological Industries (Kibbutz Beit Haemek, Israel). Dulbecco's modified Eagle's medium (DMEM) was obtained from Shanghai Yuanpei Biotechnology Corporation (Shanghai, China). SB202190, SP600125, and U0126 were purchased from Enzo (New York, USA).

\section{Animals}

APP/PS1 double-transgenic male mice and age-matched wild-type (WT) female mice with the same genetic background were originally obtained from the Model Animal Research Center of Nanjing University (Nanjing, China). Heterozygous APP/PS1 males were bred with WT females and their offspring were genotyped using PCR with primers specific for the APP-sequence (Forward: 5'-GAC TGA CCA CTC GAC CAG GTT CTG-3', Reverse: 5'-CTT GTA AGT TGG ATT CTC ATA TCC G-3') and PS1-sequence (Forward: 5'-AAT AGA GAA CGG CAG GAG CA-3', Reverse-5'-GCC ATG AGG GCA CTA ATC AT-3').

The mice were group-housed under standard laboratory conditions $\left(23 \pm 1^{\circ} \mathrm{C}, 55 \pm 5 \%\right.$ humidity, $12 \mathrm{~h}$ light/dark cycle). A standard diet and distilled water were supplied ad libitum. All experiments were carried out in strict accordance with the National Institutes of Health Guide for Care and Use of Laboratory Animals, and all procedures for animal use were approved by the Institutional Animal Care and Utilization Committee of Xi'an Jiaotong University Health Science Center.

\section{Intracerebral injection of lentiviral particles}

The lentiviruses containing short hairpin sequences (TREM2 shRNA) (mouse TREM2 gene, NCBI ID: NM_031254.3) and empty vector (as controls) were provided by Genechem Co., Ltd. (Shanghai). TREM2 was expressed under the promoter of fractalkine receptor (CX3CR1) in vivo and in vitro. The titer for lentiviral particles containing TREM2 shRNA (LV-shTREM2) was $8 \times 10^{8}$ transduction units (TU)/mL, and the titer for control lentiviral particles (LV-Con) was $2.5 \times 10^{9} \mathrm{TU} / \mathrm{mL}$. 6-month-old male APP/PS1 mice and their age and sex-matched WT mice were randomized into the WT group $(n=10)$, APP/PS1 group $(n=10)$, APP/PS1 + LV-Con group $(n=10)$, and APP/PS1 + LV-shTREM2 group $(n=10)$. Intracerebral injection of lentiviral particles was performed as previously described [6, 24]. Briefly, mice were anesthetized by intraperitoneal injection of sodium pentobarbital and placed on a stereotaxic apparatus (Shenzhen RWD Life Science Co., Ltd. China). The lentiviral particles were delivered into the cerebral cortex (two deposits) and hippocampus (one deposit) of each hemisphere at the speed of $0.5 \mu \mathrm{l} / \mathrm{min}$ using a 28-gauge stainless steel needle. The coordinates from bregma for the cortex: AP $-0.3 \mathrm{~mm}, \mathrm{ML} \pm 2 \mathrm{~mm}$, and DV $1.5 \mathrm{~mm}$; as well as $\mathrm{AP}-2 \mathrm{~mm}, \mathrm{ML} \pm 1.2 \mathrm{~mm}$, and $\mathrm{DV}-1.2 \mathrm{~mm}$. The coordinates from bregma for the 
hippocampus: AP $-2 \mathrm{~mm}, \mathrm{ML} \pm 1.2 \mathrm{~mm}$, and $\mathrm{DV}-2 \mathrm{~mm}$. To allow the solution to diffuse into the brain and to prevent its reflux, the needle was left for an additional $5 \mathrm{~min}$ after the injection. After the scalp was sutured, mice were kept on a warm pad and returned to their home cages until they awoke. Behavioral tests were performed 2 months after the lentiviral injection (Fig. 1a).

\section{Cell culture}

The immortalized murine microglial cell line BV2 retains many of the morphological and functional properties of primary microglia [34]. BV2 cells were grown in DMEM (high glucose) supplemented with $10 \% \mathrm{FBS}, 100 \mathrm{U} / \mathrm{mL}$ penicillin, and $100 \mu \mathrm{g} / \mathrm{mL}$ streptomycin. The cells were cultured under a humidified atmosphere of $95 \%$ air and $5 \% \mathrm{CO}_{2}$ at $37^{\circ} \mathrm{C}$.

\section{Preparation of $A \beta_{1-42}$ oligomers}

The toxic oligomers of $A \beta_{1-42}$ were prepared as previously described [6,35]. Briefly, lyophilized $A \beta_{1-42}$ peptide was dissolved in HFIP at a concentration of $1 \mathrm{mg} / \mathrm{mL}$, separated into aliquots in sterile microcentrifuge tubes, dried under vacuum, and stored at $-80^{\circ} \mathrm{C}$. Immediately before using, the peptide film was first dissolved in DMSO at $2 \mathrm{mM}$ and diluted with DMEM and then incubated at $4^{\circ} \mathrm{C}$ for $24 \mathrm{~h}$ to form diffusible oligomers.

\section{Cell transfection and drug treatment}

Cell transfection was conducted as previously described [6, 24]. Briefly, $1 \times 10^{4}$ BV2 cells were seeded on 6-well plates and incubated in a growth medium until $20-30 \%$ confluence. Lentiviral particles and 25xHitransG (Genechem Co., Ltd.) were mixed gently and added to the cultures according to the manufacturer's instructions. After $8 \mathrm{~h}$ incubation in transfection medium, the supernatant was discarded immediately and the cells were incubated with a fresh medium containing $10 \%$ FBS. Each transfection was performed in triplicate and repeated 2 to 3 times.

After $48 \mathrm{~h}$ of transfection, BV2 cells were pretreated with p38 inhibitor (SB202190, $10 \mu \mathrm{M})$, JNK inhibitor (SP600125, $10 \mu \mathrm{M})$, and ERK1/2 inhibitor (U0126, $10 \mu \mathrm{M})$ for $30 \mathrm{~min}$ and then incubated with $A \beta_{1-42}$ oligomers $(5 \mu \mathrm{M})$ for another $24 \mathrm{~h}$ in a serum-free culture medium.

\section{Behavioral analysis}

\section{Novel object recognition (NOR) test}

To evaluate the object recognition memory, the NOR test was performed in a square-shaped box (40 $\mathrm{cm} \times$ $40 \mathrm{~cm} \times 40 \mathrm{~cm}$ ) for 2 days as described previously [36]. In the acquisition (training) phase, the mice were exposed to two identical objects (A1 and A2) for $5 \mathrm{~min}$, and the time spent exploring each object was recorded. Twenty-four hours later, in the testing (consolidation) phase, one object (A1) was replaced by a novel object (B) of a different material, shape, and color, and the mice were allowed to freely explore the objects for $5 \mathrm{~min}$. Time spent exploring each object was defined as directing the nose to the object at a 
distance of maximum $2 \mathrm{~cm}$ and exploring it. The recognition index $(\mathrm{RI})=$ (exploring time for novel object $\mathrm{B} /$ total exploring time for object $\mathrm{B}$ and object A2) $\times 100 \%$. The box and objects after each trial were thoroughly cleaned with $70 \%$ alcohol to minimize odor cues.

\section{Y-maze test}

Spontaneous alternation in the Y-maze test, which was used to assess short-term working spatial memory and exploratory activity of mice, was performed as described before [7, 35]. The Y-maze apparatus consists of three arms at 120 $0^{\circ}$ angles (width $6 \mathrm{~cm} \times$ length $30 \mathrm{~cm} \times$ height $15 \mathrm{~cm}$ ) to each other. Each mouse was initially placed in one arm and allowed to explore the maze freely for $8 \mathrm{~min}$, and the sequence (i.e., $A C A B C$, etc.) and number of arm entries were counted. A successful alternation was defined as entries into all three arms on consecutive choices (i.e., ABC, BCA, or CAB but not ABA). The spontaneous alternation $(\%)=[($ Number of alternations $) /($ Total arm entries -2$)] \times 100 \%$. The $Y$-maze arms were cleaned with $70 \%$ ethanol between trials to minimize odor cues.

\section{Morris water maze (MWM) test}

Spatial learning and reference memory of mice was evaluated by the MWM test as previously described $[7,35]$. The apparatus consisted of a $120 \mathrm{~cm}$ diameter circular plastic pool, filled with opaque water at the depth of $35 \mathrm{~cm}$ and controlled temperature of $25 \pm 2^{\circ} \mathrm{C}$. A white $9 \mathrm{~cm}$ diameter transparent platform was located $1 \mathrm{~cm}$ below the water surface in the center of one of the four quadrants of the maze. Mice of all groups were given four trials per day for 5 consecutive days in the hidden platform acquisition training. On each training trial, the mice were allowed to swim freely for $90 \mathrm{~s}$ to find the platform. If they failed to locate the platform within $90 \mathrm{~s}$, they were gently guided to the platform and allowed to stay there for $10 \mathrm{~s}$. On the sixth day, mice were subjected to a probe test to evaluate memory retention. During this test, the platform was removed and the mice were allowed to search the maze for $90 \mathrm{~s}$. The escape latency, the number of crossing the platform area, and the time spent in each quadrant were monitored by a video tracking system (Chengdu TME Technology Company, Chengdu, China).

\section{Preparation of brain tissue}

After all the behavioral tests, 5 mice per group were randomly selected and deeply anesthetized before transcardiac perfusion with saline followed by a cold $4 \%$ paraformaldehyde (PFA). The brains were immediately removed and postfixed with $4 \%$ PFA for $24 \mathrm{~h}$, cryoprotected with $30 \%$ sucrose for $48 \mathrm{~h}$, and cut into coronal sections at a thickness of $30 \mu \mathrm{m}$ and stored at $-20^{\circ} \mathrm{C}$ for neuropathological analysis. The cortex and hippocampus of the remaining mice were immediately isolated on an ice glass plate and then stored at $-80^{\circ} \mathrm{C}$ for subsequent biochemical analysis.

\section{Th-S staining}

To detect dense-core amyloid plaques, Th-S staining was performed as previously described [35, 36]. Briefly, the free-floating sections were washed with phosphate buffered saline (PBS, pH 7.4) three times, and then were briefly rinsed in distilled water. Next, the sections were incubated in freshly prepared $1 \%$ Th$S$ aqueous solution at room temperature in the dark for $5 \mathrm{~min}$ followed by differentiation using $70 \%$ 
ethanol for $1 \mathrm{~min}$. Finally, the sections were covered with aqueous mounting media and examined by the fluorescence microscope. A $\beta$ plaques number and Th-S staining area (plaque area/total area selected $x$ $100 \%$ ) were quantified in five fields of each section (6 sections per mouse) using Image-Pro Plus 6.0 software.

\section{Immunohistochemistry and immunofluorescence}

Immunohistochemistry staining was carried out as previously described [35, 36]. Briefly, free-floating sections were washed with PBS, pre-treated in $3 \%$ hydrogen peroxide, and maintained in blocking solution (1\% BSA and $0.3 \%$ Triton $\mathrm{X}-100)$ for $1 \mathrm{~h}$ at room temperature. Subsequently, sections were incubated overnight at $4^{\circ} \mathrm{C}$ with primary antibodies diluted in blocking solution (anti-lba1: 1/900; anti-

NeuN:1/10000). After washing with PBS, the sections were reacted with secondary antibody at $37^{\circ} \mathrm{C}$ for 2 h, and visualized using a 3, 3'-diaminobenzidine (DAB) substrate kit. Finally, the sections were mounted with neutral balsam and examined by the light microscopy. For immunofluorescence staining, the sections were incubated overnight at $4^{\circ} \mathrm{C}$ with primary antibodies diluted in blocking solution (anti-GFAP: 1/5000; anti-lba1: 1/900; anti-MOAB2:1/2000), followed by incubation with Cy3 or FITC-conjugated secondary antibody at $37^{\circ} \mathrm{C}$ for $2 \mathrm{~h}$. After washing with PBS, the sections were counterstained with DAPI and visualized by the fluorescent microscope. Six random visual fields of the cortex and hippocampus were photographed in each section, and the number of staining cells in each field was counted by a researcher who was blind to the condition with Image-Pro Plus 6.0 software.

\section{Quantitative real-time reverse transcription PCR (qRT-PCR)}

Total RNA was extracted from the cortex, hippocampus, and BV2 cells with RNAiso Plus reagent. An equal amount of total RNA (500 ng) was reverse transcribed into cDNA in a $10 \mu \mathrm{L}$ reaction volume according to the manufacturer's instructions. qRT-PCR was carried out in 96-well plates on a fluorescence thermocycler iQ5 (Bio-Rad) with initial denaturation at $95^{\circ} \mathrm{C}$ for $30 \mathrm{~s}$ followed by 40 cycles of amplification $\left(95^{\circ} \mathrm{C}\right.$ for $10 \mathrm{~s}, 60^{\circ} \mathrm{C}$ for $30 \mathrm{~s}$, and $72^{\circ} \mathrm{C}$ for $\left.30 \mathrm{~s}\right)$. The primer sequences used in the current experiment are as follows: 5'-CCT GAA GAA GCG GAA TGG-3' and 5'-GGA GAC TCT TGA CAC TGG TA-3' for TREM2; 5'-GTC TAC TGA ACT TCG GGG TGA T-3' and 5'-ATG ATC TGA GTG TGA GGG TCT G-3' for TNF-a; 5'-GAA GAG CCC ATC CTC TGT GA-3' and 5'-ATG ATC TGA GTG TGA GGG TCT G -3' for IL-1 3 ; 5'ACA AAG CCA GAG TCC TTC AGA G-3' and 5'-CAT TGG AAA TTG GGG TAG GA-3' for IL-6; and 5'-ACC ACA CCT TCT ACA ATG AG-3' and 5'-GGT TGG TGA AGT TGG TAG G-3' for $\beta$-actin. $\beta$-actin was used as an internal control for normalization. Relative gene expression was quantified using the $2^{-\Delta \Delta C t}$ method. To detect the efficiency of lentiviral-mediated downregulation of TREM2 expression, the PCR products were separated by electrophoresis in $1.5 \%$ agarose gels and stained with ethidium bromide.

\section{ELISA assay}

ELISA assays for $A \beta_{1-40}, A \beta_{1-42}$, and pro-inflammatory cytokines levels were described previously [7, 35]. In brief, cortical and hippocampal tissues of each mouse were homogenized in cold homogenization buffer containing protease inhibitor mixtures. Homogenates were centrifuged at $15,000 \mathrm{rpm}$ for $1 \mathrm{~h}$ at $4^{\circ} \mathrm{C}$, and the supernatants (soluble fraction) were collected. The acquired pellet was incubated with guanidine 
buffer containing protease inhibitor mixtures for $2 \mathrm{~h}$ at room temperature and further centrifuged as above [37]. The supernatants were collected and used as an insoluble fraction. BV2 cells were seeded in 6-well culture plates. After treatment, the supernatants were collected and centrifuged at 8,000 rpm for 30 min at $4^{\circ} \mathrm{C}$ to remove cell debris. The supernatants were assayed by ELISA for soluble/insoluble $A \beta_{1-40}$ and $A \beta_{1-42}, T N F-a, I L-1 \beta$, and IL-6 according to the manufacturer's instructions. Absorbance measurements were read at $450 \mathrm{~nm}$ with a microplate reader.

\section{Western blotting}

Western blotting was performed as described previously [38]. Briefly, the brain tissues and BV2 cells were homogenized in ice-cold lysis buffer containing protease inhibitor mixtures. After centrifugation, the levels of protein in the supernatant were measured using the BCA protein assay kit. The lysates $(20 \mu \mathrm{g})$ were boiled and separated by $10 \%$ sodium dodecyl sulfate-polyacrylamide gel electrophoresis and then transferred onto NC membranes. Membranes were blocked and incubated overnight at $4^{\circ} \mathrm{C}$ with primary antibodies including TREM2, Iba1, GFAP, TLR4, MyD88, TRAF6, phospho-p38, total-p38, phospho-JNK, total-JNK, phospho-ERK1/2, total-ERK1/2, Syn, PSD-95, and $\beta$-actin. After washing, the membranes were incubated with the appropriate secondary antibodies and visualized with the ECL detection reagents. Western blotting results were analyzed using the Image $\mathrm{J}$ software.

\section{Statistical analysis}

Data were expressed as mean \pm standard deviation (SD). The individual experiment was carried out at least three times. All statistical analyses were performed using the SPSS 13.0 software. Group differences in the escape latency in the MWM test were analyzed using two-way analysis of variance (ANOVA) with repeated measures. The other data were analyzed using one-way ANOVA followed by Tukey's post-hoc analysis. Statistical significance was considered at $p<0.05$.

\section{Results}

\section{TREM2 is upregulated in APP/PS1 mice and is localized mainly on microglia}

First, double immunofluorescence staining was performed to determine the cellular localization of TREM2 in the brain of 5-month-old APP/PS1 mice. The results showed a good co-localization of TREM2 and microglia marker Iba1 in the brain as we recently reported [24] (Fig. 1b). Afterward, qRT-PCR and western blotting were performed to examine the dynamic changes of TREM2 expression in APP/PS1 mice at 1, 3, 5, and 7 months of age. As revealed in Fig. 1c, TREM2 mRNA levels in the cortex and hippocampus of APP/PS1 mice were significantly increased at 3 months, and gradually increased from 3 to 7 months. Simultaneously, TREM2 protein levels in the cortex and hippocampus of APP/PS1 mice showed a similar trend during disease progression (Fig. 1d). 


\section{Downregulation of TREM2 expression exacerbated A $\beta$ deposition in APP/PS1 mice}

To further explore the effects of TREM2 on learning and memory ability and AD-related pathophysiologic changes, a lentiviral-mediated strategy was performed to downregulate TREM2 expression in the brain of APP/PS1 mice. TREM2 mRNA levels in the cortex and hippocampus were successfully reduced by $53.06 \%$ and $40.23 \%$ respectively, at 2 months after injection of lentiviral particles (Fig. S1a). Similarly, TREM2 protein levels in the cortex and hippocampus were effectively decreased by $46.15 \%$ and $35.71 \%$ respectively (Fig. S1b).

Amyloid plaques derived from $A \beta$ deposition are one of the most significant characteristics in the development of $A D$. Th-S staining was used to determine the amyloid plaques in the brain. The deposition of $A \beta$ in the brain of APP/PS1 mice was much more severe after downregulation of TREM2 expression (Fig. 2a). The percentages of the number of $A \beta$ plaques (number per $\mathrm{mm}^{2}$ ) were markedly increased by $50.41 \%$ and $55.8 \%$ respectively, compared to the APP/PS1 group (Fig. 2b). Meanwhile, the Th-S staining area in the cortex and hippocampus showed a similar trend (Fig. 2c). A $\beta$ levels in the brain tissue which were paralleled with $A \beta$ deposition were further detected by ELISA. As expected, higher levels of soluble $A \beta_{1-40}$ and $A \beta_{1-42}$ in the cortex and hippocampus were observed in APP/PS1 mice that received TREM2 lentivirus (Fig. 2d, e). Simultaneously, insoluble $A \beta_{1-40}$ and $A \beta_{1-42}$ in the cortex and hippocampus were also dramatically increased (Fig. $2 f, g$ ). Overall, these data indicated that $A \beta$ plaque burden was significantly elevated by the downregulation of TREM2 expression in APP/PS1 mice.

\section{Downregulation of TREM2 expression induced microgliosis and astrogliosis in APP/PS1 mice}

Reactive microglia and astrocytes surrounding $A \beta$ plaques is an early feature of $A D$ pathogenesis, which may trigger neuroinflammation and further amplifying neuronal damage. To explore whether downregulation of TREM2 expression could induce widespread and striking microgliosis and astrogliosis, immunohistochemistry, immunofluorescence, and western blotting were performed. The number of Iba1positive microglia and GFAP-positive astrocytes were significantly increased in the brain of APP/PS1 mice that received TREM2 lentivirus (Fig. 3a, b). Furthermore, Iba1 and GFAP protein levels were also markedly elevated in the cortex (Fig. 3c) and hippocampus (Fig. 3d) in these mice. As expected, large numbers of Iba1-positive reactive microglia and GFAP-positive reactive astrocytes were distributed around the more $A \beta$ plaques in both the cortex and hippocampus of APP/PS1 mice after downregulation of TREM2 expression as assessed by double staining of Iba1/AB (MOAB2) plaques (Fig. 4) and GFAP/A $\beta$ (MOAB2) plaques (Fig. 5). These data support the notion that TREM2 downregulation activated microglia and astrocytes, which subsequently induced widespread and striking microgliosis and astrogliosis in the brain of APP/PS1 mice.

\section{Downregulation of TREM2 expression promoted pro- inflammatory cytokines production in APP/PS1 mice}


Activated microglia and astrocytes stimulated by $A \beta$ may be involved in the acceleration of the AD pathological process by releasing pro-inflammatory cytokines and other toxic mediators, which leads to an increase in $A \beta$ production and accumulation, neuronal damage, as well as cognitive impairment [3941]. To examine the potential role of TREM 2 on the production of pro-inflammatory cytokines, we first investigated the mRNA levels of TNF- $\alpha$, IL-1 13 , and IL- 6 in the cortex and hippocampus. As shown in Fig. $6 a$, the mRNA levels of TNF- $a$, IL-1 $\beta$, and IL- 6 were significantly increased in the brain of APP/PS1 mice that received TREM2 lentivirus. We next evaluated the protein levels of TNF- $a, \mathrm{IL}-1 \beta$, and IL- 6 by ELISA to further confirm the above results. Similarly, corresponding increments in protein levels of TNF-a, IL-1 $\beta$, and IL-6 in the cortex and hippocampus were also observed (Fig. 6b). Collectively, these findings strongly suggested that TREM2 downregulation enhanced the secretion and production of proinflammatory cytokines released by activated glial cells in APP/PS1 mice.

\section{Downregulation of TREM2 expression enhanced neuroinflammatory responses via TLR4-mediated MAPK signaling pathway in APP/PS1 mice}

After binding to $A \beta$, TLR4 recruits the pivotal downstream signaling adaptor molecules MyD88 and TRAF6, activating multiple downstream signaling molecules, including the MAPK family. It is well established that MAPK signaling pathway activation is critical for the production of various proinflammatory cytokines and mediators [6, 42]. To demonstrate that downregulation of TREM2 expression can aggravate neuroinflammation via TLR4-mediated MAPK signaling pathway. We first measured the expression of TLR4/MyD88 signaling cascade by western blotting. As revealed by Fig. 7a, b, TLR4, MyD88, and TRAF6 were dramatically increased in the cortex and hippocampus of APP/PS1 mice that received TREM2 lentivirus. Furthermore, we examined the activation of MAPK signaling proteins. Downregulation of TREM2 expression effectively enhanced phospho-p38, JNK, and ERK1/2 in the cortex (Fig. 7c) and hippocampus (Fig. 7d) of APP/PS1 mice. Therefore, these findings suggested that TREM2 downregulation regulates TLR4-mediated activation of MAPK signaling pathway.

\section{Downregulation of TREM2 expression promoted neuronal and synaptic loss in APP/PS1 mice}

The synaptic dysfunction and neuronal loss in the hippocampus and association cortices, is widely considered as the main contributors to cognitive deficits in AD [43-45]. Therefore, we explored whether downregulation of TREM2 expression can exacerbate neurodegenerative changes in APP/PS1 mice. First, the number of NeuN-positive neurons was explored by immunohistochemical staining. As revealed by Fig. 8a, the number of NeuN-positive neurons was significantly decreased in the cortex and hippocampal cornu ammonis 1 (CA1) region of APP/PS1 mice that received TREM2 lentivirus. Next, the synapticassociated proteins Syn and PSD-95 were examined by western blotting. As anticipated, downregulation of TREM2 expression markedly decreased the levels of pre-synaptic protein Syn and post-synaptic protein PSD-95 in the cortex (Fig. 8b) and hippocampus (Fig. 8c). Collectively, these findings indicated that TREM2 downregulation increases neuronal and synaptic loss in APP/PS1 mice. 


\section{Downregulation of TREM2 expression exacerbated learning and memory deficits in APP/PS1 mice}

To demonstrate downregulation of TREM2 expression could aggravate behavioral deficits in APP/PS1 mice, NOR test, Y-maze test, and MWM test were conducted. The NOR task is gaining popularity for its ability to evaluate the non-spatial memory and the preference for novelty in rodents (Fig. 9a). As shown in Fig. 9b, no significant differences were observed in the time exploring two identical objects during the acquisition phase, which suggested that the experimental groups were equally motivated to explore the objects. After $24 \mathrm{~h}$, in the testing phase with two different objects, RI was dramatically decreased in APP/PS1 mice compared to WT mice. Importantly, a more obvious deficit in exploring the novel object was found in APP/PS1 mice that received TREM2 lentivirus (Fig. 9c). After that, we investigated alterations of hippocampal-dependent spatial memory by measuring Y-maze spontaneous alternation. In contrast to WT mice, APP/PS1 mice displayed a reduced percentage of spontaneous alternation. Interestingly, this decreased spontaneous alternation behavior was effectively exacerbated by downregulation of TREM2 expression (Fig. 9d). Finally, the MWM test was performed to evaluate the ability of spatial learning and long-term memory of mice. In the hidden platform test, all mice gradually learned the location of the platform as training progressed, and the escape latency fell to the shortest on the fifth day. As anticipated, a longer escape latency was observed in APP/PS1 mice that received TREM2 lentivirus (Fig. 9e, f). Moreover, APP/PS1 mice that received TREM2 lentivirus showed a fewer number of platform traverses and a lesser percentage of time spent in the target quadrant, implying an obvious spatial memory impairment (Fig. 9g, h).

\section{Knockdown of TREM2 expression increased pro- inflammatory cytokines production in $A \beta_{1-42}$-induced BV2 cells}

To further assess the potential regulatory effects of TREM2 on neuroinflammation induced by oligomeric $A \beta_{1-42}$ in microglia, a lentiviral-mediated strategy was used to knock down TREM2 expression in BV2 cells. The expression levels of TREM2 were validated using qRT-PCR at $48 \mathrm{~h}$ and western blotting at $72 \mathrm{~h}$ after transfection, respectively. As revealed by Fig. S2a, the mRNA levels of TREM2 significantly decreased by nearly $54.35 \%$ compared with control. Similarly, the protein levels of TREM2 markedly decreased by nearly $27.94 \%$ (Fig. S1b).

After transfection for $48 \mathrm{~h}, \mathrm{BV} 2$ cells were cultured with $5 \mu \mathrm{M}$ oligomeric $A \beta_{1-42}$ for another $24 \mathrm{~h}$, and the expression levels of pro-inflammatory cytokines were examined. As indicated by Fig. 10a, the mRNA levels of TNF- $\alpha$, IL-1 $\beta$, and IL- 6 were dramatically increased upon $A \beta_{1-42}$ stimulation. Interestingly, knockdown of TREM2 expression enhanced the $A \beta_{1-42}$-induced inflammatory responses, as the mRNA levels of TNF- $\alpha$, IL-1 $\beta$, and IL- 6 were further increased. Similarly, the protein levels of TNF- $\alpha$, IL-1 $\beta$, and IL- 6 in the media were consistent with the mRNA levels (Fig. 10b). 


\section{Knockdown of TREM2 expression aggravated $A \beta_{1-42^{-}}$ induced inflammatory responses via TLR4-mediated MAPK signaling pathway in BV2 cells}

TLR4 induces inflammatory responses mediated by the MAPK signaling pathway, which plays a key role in controlling the production of pro-inflammatory cytokines [6, 42]. To determine whether knockdown of TREM2 expression aggravates $A \beta_{1-42}$-induced inflammatory responses through the TLR4/MAPK signaling pathway, BV2 cells were transfected with lentiviral particles for $48 \mathrm{~h}$, followed by $5 \mu \mathrm{M}$ oligomeric $A \beta_{1-42}$ for another $24 \mathrm{~h}$, the protein levels of TLR4, MyD88, TRAF6, p38, JNK, and ERK1/2 were examined by western blotting. As demonstrated by Fig. 11a, TLR4 and its downstream proteins MyD88 and TRAF6 were markedly upregulated following $A \beta_{1-42}$ treatment in BV2 cells. TREM2 knockdown further increased the expression of TLR4, MyD88, and TRAF6. After stimulation with $A \beta_{1-42}$, the phosphorylation of p38, JNK, and ERK1/2 was significantly increased. Importantly, TREM2 knockdown further elevated the phosphorylation of $\mathrm{p} 38, \mathrm{JNK}$, and ERK1/2 in BV2 cells (Fig. 11b). These results indicated that TREM2 knockdown regulates $A \beta_{1-42}$-induced neuroinflammation by activation of TLR4/MAPK signaling pathway.

\section{Blockade of MAPK inhibited $A \beta_{1-42}$-induced pro- inflammatory cytokines production in TREM2-knocked down BV2 cells}

To investigate whether knockdown of TREM2 expression plays an important role in regulating $A \beta_{1-42^{-}}$ induced pro-inflammatory cytokines production via the MAPK signaling pathway, BV2 cells were transfected with lentiviral particles for $48 \mathrm{~h}$, then pretreated with $10 \mu \mathrm{M}$ SB202190 (p38 specific inhibitor) , $10 \mu \mathrm{M}$ SP600125 (JNK specific inhibitor), or $10 \mu \mathrm{M}$ U0126 (ERK1/2 specific inhibitor) for $30 \mathrm{~min}$, followed by $5 \mu \mathrm{M}$ oligomeric $A \beta_{1-42}$ for another $24 \mathrm{~h}$. As shown in Fig. 12a, the mRNA levels of TNF- $a$, IL$1 \beta$, and IL- 6 were increased upon knockdown of TREM2 expression. However, the presence of SB202190, SP600125, and U0126 eliminated the increased mRNA levels of TNF- $\alpha$, IL-1 $\beta$, and IL- 6 in BV2 cells resulted from TREM2 down-regulation. Meanwhile, the protein levels of TNF-a, IL-1 $\beta$, and IL- 6 in the media were also increased upon TREM2 knockdown in BV2 cells. Likewise, these inhibitors ameliorated the higher protein levels of TNF-a, IL-1 $\beta$, and IL- 6 in the media (Fig. 12b). From the above results, we concluded that TREM2 regulates pro-inflammatory cytokines production by the MAPK signaling pathway.

\section{Discussion}

In the current study, to explore the effects and the molecular mechanism of TREM2 on A $\beta$ pathology, neuroinflammatory responses, neurodegenerative changes, and behavioral deficits, we used a lentiviralmediated strategy to downregulation of TREM2 expression in the brain of APP/PS1 mice and BV2 cells. Our results demonstrated that TREM2 was uniquely expressed by microglia, and the expression of 
TREM2 was significantly increased with aging in the cortex and hippocampus of APP/PS1 mice. Furthermore, downregulation of TREM2 expression exacerbated neuropathologies including $A \beta$ deposition, microgliosis, astrogliosis, as well as neuronal and synaptic loss, which was accompanied by a decline in cognitive function in APP/PS1 mice. Moreover, the mechanistic study revealed that downregulation of TREM2 expression increases pro-inflammatory cytokines production via TLR4mediated MAPK signaling pathway in the brain of APP/PS1 mice and BV2 cells. These data coupled with the previous findings strongly suggested that TREM2 is involved in AD pathogenesis, and it may represent a potential therapeutic target against $\operatorname{AD}[20,24,29]$.

Neuroinflammation is a critical pathological feature and considered a major contributor to AD pathogenesis [1]. Activated microglia and astrocytes are both involved in the initialization and progression of neuroinflammation in $A D$. There is increasing evidence to suggest that neuroinflammatory responses were closely associated with the several $A D$-related pathological processes, including $A B$ accumulation, neuronal and synaptic loss, tau pathology, as well as cognitive deficits [39-41]. In the present study, after downregulation of TREM2 expression, the number of Iba1-positive microglia and GFAP-positive astrocytes were markedly increased, and they clustered around A $\beta$ plaques. Furthermore, the protein levels of Iba1 and GFAP were effectively elevated in the cortex and hippocampus of APP/PS1 mice. The current study indicated that TREM2 downregulation leads to more obvious microgliosis and astrogliosis in the brain of APP/PS1 mice, which are closely associated with neuroinflammation. However, the molecular mechanisms by which TREM2 downregulation aggravated neuroinflammatory responses remains unclear. TLR4, a pattern recognition receptor for $A \beta$, is highly expressed on microglia and astrocytes surfaces. TLR4 ligation by $A \beta$ can activate multiple downstream signaling pathways, including MAPK. In line with this notion, the expression of TLR4/MAPK signaling pathway molecules was determined. The present study revealed that TLR4, MyD88, and its adapter protein TRAF6 were markedly upregulated in the brain of APP/PS1 mice after downregulation of TREM2 expression. Furthermore, the phosphorylation of $\mathrm{p} 38, \mathrm{JNK}$, and ERK1/2 was significantly increased in the cortex and hippocampus of APP/PS1 mice. In A $\beta_{1-42}$-induced BV2 cells, we found that TREM2 downregulation significantly elevated the levels of TLR4, MyD88, and TRAF6. Meanwhile, a significant increase of phospho-p38, JNK, and ERK1/2 were also observed after knockdown of TREM2 expression. Taken together with previous findings, our results suggested that TREM2 downregulation aggravated neuroinflammatory responses through TLR4/MAPK signaling pathway in AD [46].

Mounting evidence suggests that $A \beta$ induces TLR4/MAPK signaling pathway activation, which subsequently stimulates the activated microglia and astrocytes to produce and release pro-inflammatory cytokines and other inflammatory mediators.

TNF- $a$ can stimulate $\beta$ - and $y$-secretase enzyme activity, which results in increased synthesis of $A \beta$ peptides and a further increase in TNF-a release $[10,47]$. This auto-amplified loop in the AD brain can contribute to the maintenance of excessive levels of TNF- $a$, which then stimulate $A \beta$ synthesis and neuronal loss, also suppressing microglia phagocytosis of $A \beta[47,48]$. Besides, TNF- $\alpha$ could contribute to promoting insulin resistance and finally lead to cognitive decline in $\operatorname{AD}[49,50]$. IL-1 $\beta$ activates p38 
pathway, which could lead to tau hyperphosphorylation and further exacerbate synaptic and neuronal cell dysfunction $[9,51]$. Similarly to TNF- $a, I L-1 \beta$ can promote $A \beta$ production by modulating $Y$-secretase enzyme activity in neurons [10]. IL-6 stimulates and promotes the recruitment of microglia and astrocytes to release pro-inflammatory cytokines, and it also promotes $\beta$-secretase enzyme activity and tau phosphorylation in $\operatorname{AD}[11,52,53]$. Our results showed that pro-inflammatory cytokines TNF-a, IL-1 $\beta$, and IL-6 were increased in the cortex and hippocampus of APP/PS1 mice after downregulation of TREM2 expression. Furthermore, knockdown of TREM2 expression also elevated the production of TNF-a, IL-1 $\beta$, and IL-6 in BV2 cells, similar to the results of in vivo studies. The current findings were coincident with previous reports, which identified that downregulation of TREM2 expression may contribute to the development of neuroinflammation in $A D[27,28]$. Afterward, we attempted to elucidate whether knockdown of TREM2 expression could regulate $A \beta_{1-42}$-induced neuroinflammatory responses via the MAPK signaling pathway. We treated TREM2-knocked down BV2 cells with the p38 inhibitor SB202190, JNK inhibitor SP600125, and ERK1/2 inhibitor U0126. The current results showed that the higher levels of pro-inflammatory cytokines TNF-a, IL-1 $\beta$, and IL-6 seen in TREM2 knockdown BV2 cells were significantly suppressed by SB202190, SP600125, and U0126. Collectively, these findings demonstrated that TREM2 regulates pro-inflammatory cytokines production by the MAPK signaling pathway.

Previous studies reported that neuroinflammation is related to the aggregation of $A \beta$ in the brain of $A D$ $[54,55]$. Abnormal A $\beta$ deposition can stimulate nuclear factor-KB (NF-KB) and MAPK signaling pathways, which are associated with the transcription of pro-inflammatory cytokines and mediators. In turn, proinflammatory cytokines and mediators cause the initiation of APP cleavage through the MAPK and NF-KB signaling pathways $[10,56]$. Therefore, $A \beta$ can be viewed both as a cause and consequence of neuroinflammation in AD. TREM2 facilitates microglia clustering around $A \beta$ plaques and promotes $A \beta$ phagocytosis and degradation. However, TREM2 deletion decreased the microglial phagocytic clearance of $A \beta$, resulting in exacerbated brain $A \beta$ deposition and cognitive deficits in the $A D$ animal model $[22,57]$. In this study, after downregulation of TREM2 expression, more $A \beta$ plaques in the cortex and hippocampus of APP/PS1 mice were observed. Likewise, the protein levels of both soluble/insoluble $A \beta_{1-40}$ and $A \beta_{1-42}$ were higher in the brain of APP/PS1 mice after TREM2 downregulation. Thus, combined with previous reports, downregulation of TREM2 expression may lead to $A \beta$ aggregation and accumulation in the brain, and further exacerbating spatial cognitive impairment $[27,28,58]$.

Both activated microglia and astrocytes may gradually release pro-inflammatory cytokines TNF- $\alpha$, IL-1 and IL-6. These cytokines are destructive to neurons by altering synaptic proteins and finally lead to cognitive deficits $[59,60]$. The synapse-associated proteins, especially pre-synaptic Syn and post-synaptic PSD-95, play important roles in synaptic plasticity and memory formation $[61,62]$. It has been shown that deficits in Syn and PSD-95 correlate with cognitive impairment in AD and aging $[63,64]$. Previous studies reported that there is a direct interaction between neuroinflammation and Syn as well as PSD-95 in newborn neurons $[65,66]$. In this study, TREM2 downregulation remarkably increased neuronal loss in the cortex and hippocampal CA1 region of APP/PS1 mice. Additionally, the levels of Syn and PSD-95 markedly decreased in the cortex and hippocampus of APP/PS1 mice after downregulation of TREM2 
expression. These data showed that TREM2 downregulation led to the exacerbation of neurodegenerative changes, as the more severe neuronal and synaptic loss was observed. Cognitive dysfunction is associated with neuronal and synaptic loss in the cortex and hippocampus in $\operatorname{AD}[27,67,68]$. Thus, we assessed the learning and memory capacity of APP/PS1 mice using established behavioral tests. In the NOR test, APP/PS1 mice exhibited a significantly lower preference for exploring the novel object in the testing phase following TREM2 downregulation, indicating the recognition memory impairment. Furthermore, short-term working memory impairment was evaluated by the Y-maze test. APP/PS1 mice showed a markedly reduced percentage of spontaneous alteration behavior after downregulation of TREM2 expression. At last, in the hidden platform test, the escape latency was increased in APP/PS1 mice that received TREM2 lentivirus. When removing the hidden platform, APP/PS1 mice had a significant decrease in the number of target crossing and the time spent in the target quadrant, suggesting an obvious spatial memory disorder. Our study coupled with the work of others strongly revealed that exacerbation of neuronal and synaptic loss may contribute to worsening cognitive deficits after downregulation of TREM2 expression [22, 27, 57].

In conclusion, this study revealed that downregulation of TREM2 expression in the brain of APP/PS1 mice markedly exacerbated AD-related neuropathology including $A \beta$ deposition, gliosis, neuroinflammation, as well as neuronal and synaptic loss, which was accompanied by a decline in cognitive function in APP/PS1 mice. Furthermore, in vivo and in vitro evidence demonstrated that TREM2 downregulation increases the production of pro-inflammatory cytokines through TLR4-mediated MAPK signaling pathway activation. Taken together, our results indicated that TREM2 could be a promising treatment to improve cognitive deficits and neuroinflammation in $A D$ and other central nervous system diseases.

\section{Declarations}

\section{Author Contributions}

WNY, JBR, XQP and YYH designed the research, conducted the majority of the experiments, interpreted the results, and wrote the paper. XYW, QZZ, BD and CHL conducted the behavior experiments and interpreted the results. WNY is responsible for the supervision and the acquisition of funding. All authors read and approved the final manuscript.

\section{Funding Information}

This work was supported by the Natural Science Basic Research Plan in Shaanxi Province of China (2018JM7056); China Postdoctoral Science Foundation (2017T100758, 2016M590955); and Natural Science Foundation of China (81500928).

\section{Data Availability}


The datasets supporting the conclusions of this article are included within the article (and its additional file).

\section{Compliance with Ethical Standards}

All animal experiments were approved by the Institutional Animal Care and Use Committee of Xi'an Jiaotong University.

\section{Conflict of Interest}

The authors declare that there are no conflicts of interest.

\section{Consent for Participate}

Not applicable.

\section{Consent for Publication}

Not applicable.

\section{References}

1. Salinaro AT, Pennisi M, Di Paola R, Scuto M, Crupi R, Cambria MT, Ontario ML, Tomasello M et al (2018) Neuroinflammation and neurohormesis in the pathogenesis of Alzheimer's disease and Alzheimer-linked pathologies: modulation by nutritional mushrooms. Immun Ageing 15, 8.

2. Woo JH, Lee JH, Kim H, Park SJ, Joe EH, Jou I (2015) Control of inflammatory responses: a new paradigm for the treatment of chronic neuronal diseases. Exp Neurobiol 24, 95-102.

3. Morales I, Guzman-Martinez L, Cerda-Troncoso C, Farias GA, Maccioni RB (2014) Neuroinflammation in the pathogenesis of Alzheimer's disease. A rational framework for the search of novel therapeutic approaches. Front Cell Neurosci 8, 9.

4. Sarlus H, Heneka MT (2017) Microglia in Alzheimer's disease. J Clin Invest 127, 3240-3249.

5. Zhou J, Yu W, Zhang M, Tian X, Li Y, Lv Y (2019) Imbalance of microglial TLR4/TREM2 in LPStreated APP/PS1 transgenic mice: a potential link between Alzheimer's disease and systemic inflammation. Neurochem Res 44, 1138-1151.

6. He YY, Ruganzu JB, Jin H, Peng XQ, Ji SF, Ma YB, Zheng LM, Yang WN (2020) LRP1 knockdown aggravates Abeta1-42-stimulated microglial and astrocytic neuroinflammatory responses by modulating TLR4/NF-kappaB/MAPKs signaling pathways. Exp Cell Res 394, 112166.

7. He YY, Ruganzu JB, Zheng QZ, Wu XY, Jin H, Peng XQ, Ding B, Lin CH et al (2020) Silencing of LRP1 exacerbates inflammatory response via TLR4/NF-kappaB/MAPKs signaling pathways in APP/PS1 
transgenic mice. Mol Neurobiol 57, 3727-3743.

8. Martinez FO, Helming L, Gordon S (2009) Alternative activation of macrophages: an immunologic functional perspective. Annu Rev Immunol 27, 451-483.

9. Johnstone M, Bennett N, Standifer C, Smith A, Han A, Bettaieb A, Whelan J, Donohoe DR (2017) Characterization of the pro-inflammatory cytokine il-beta 1 on butyrate oxidation in colorectal cancer cells. J Cell Biochem 118, 1614-1621.

10. Liao YF, Wang BJ, Cheng HT, Kuo LH, Wolfe MS (2004). Tumor necrosis factor-alpha, interleukin-1 beta, and interferon-gamma stimulate gamma-secretase-mediated cleavage of amyloid precursor protein through a JNK-dependent MAPK pathway. J Biol Chem 279, 49523-49532.

11. Querfurth HW, LaFerla FM (2010) Mechanisms of disease Alzheimer's disease. N Engl J Med 362, 329-344.

12. Ulrich JD, Ulland TK, Colonna M, Holtzman DM (2017) Elucidating the role of TREM2 in Alzheimer's disease. Neuron 94, 237-248.

13. Hickman SE, El Khoury J (2014). TREM2 and the neuroimmunology of Alzheimer's disease. Biochem Pharmacol 88, 495-498.

14. Ma L, Allen M, Sakae N, Ertekin Taner N, Graff Radford NR, Dickson DW, Younkin SG, Sevlever D (2016) Expression and processing analyses of wild type and p.R47H TREM2 variant in Alzheimer's disease brains. Mol Neurodegener 11,72.

15. Jay TR, Hirsch AM, Broihier ML, Miller CM, Neilson LE, Ransohoff RM, Lamb BT, Landreth GE (2017) Disease progression-dependent effects of TREM2 deficiency in a mouse model of Alzheimer's disease. J Neurosci 37, 637-647.

16. Srinivasan K, Friedman BA, Larson JL, Lauffer BE, Goldstein LD, Appling LL, Borneo J, Poon C et al (2016) Untangling the brain's neuroinflammatory and neurodegenerative transcriptional responses. Nat Commun 7, 11295.

17. Tanzi RE (2013) A brief history of Alzheimer's disease gene discovery. J Alzheimer Dis 33, S5-S13.

18. Gratuze M, Leyns CEG, Holtzman DM (2018) New insights into the role of TREM2 in Alzheimer's disease. Mol Neurodegener 13,66.

19. Ulland TK, Song WM, Huang SCC, Ulrich JD, Sergushichev A, Beatty WL, Loboda AA, Zhou Y et al (2017) TREM2 maintains microglial metabolic fitness in Alzheimer's disease. Cell 170, 649-663.

20. Jiang T, Tan L, Zhu XC, Zhang QQ, Cao L, Tan MS, Gus LZ, Wang HF et al (2014) Upregulation of TREM2 ameliorates neuropathology and rescues spatial cognitive impairment in a transgenic mouse model of Alzheimer's disease. Neuropsychopharmacology 39, 2949-2962.

21. Melchior B, Garcia AE, Hsiung BK, Lo KM, Doose JM, Thrash JC, Stalder AK et al (2010) Dual induction of TREM2 and tolerance-related transcript, Tmem176b, in amyloid transgenic mice: implications for vaccine-based therapies for Alzheimer's disease. Asn Neuro 2, 157-170. 
22. Wang Y, Cella M, Mallinson K, Ulrich JD, Young KL, Robinette ML, Gilfillan S, Krishnan GM et al (2015) TREM2 lipid sensing sustains the microglial response in an Alzheimer's disease model. Cell 160, 1061-1071.

23. Mecca C, Giambanco I, Donato R, Arcuri C (2018) Microglia and aging: the role of the TREM2-DAP12 and CX3CL1-CX3CR1 axes. Int J Mol Sci 19, 318.

24. Ruganzu JB, Zheng QZ, Wu XY, He YY, Peng XQ, Jin H, Zhou JS, Ma RY et al (2021) TREM2 overexpression rescues cognitive deficits in APP/PS1 transgenic mice by reducing neuroinflammation via the JAK/STAT/SOCS signaling pathway. Exp Neurol 336, 113506.

25. Takahashi K, Rochford CDP, Neumann H (2005) Clearance of apoptotic neurons without inflammation by microglial triggering receptor expressed on myeloid cells-2. J Exp Med 201, 647-657.

26. Zheng H, Liu CC, Atagi Y, Chen XF, Jia L, Yang L, He W, Zhang X et al (2016) Opposing roles of the triggering receptor expressed on myeloid cells 2 and triggering receptor expressed on myeloid cellslike transcript 2 in microglia activation. Neurobiol Aging 42, 132-141.

27. Jiang T, Tan L, Zhu XC, Zhou JS, Cao L, Tan MS, Wang HF, Chen Q et al (2015) Silencing of TREM2 exacerbates tau pathology, neurodegenerative changes, and spatial learning deficits in P301S tau transgenic mice. Neurobiol Aging 36, 3176-3186.

28. Jiang T, Yu JT, Zhu XC, Tan MS, Gu LZ, Zhang YD, Tan L (2014) Triggering receptor expressed on myeloid cells 2 knockdown exacerbates aging-related neuroinflammation and cognitive deficiency in senescence-accelerated mouse prone 8 mice. Neurobiol Aging 35, 1243-1251.

29. Jiang T, Zhang YD, Chen Q, Gao Q, Zhu XC, Zhou JS, Shi JQ, Lu H et al (2016). TREM2 modifies microglial phenotype and provides neuroprotection in P301S tau transgenic mice. Neuropharmacology 105, 196-206.

30. Zhong L, Chen XF, Zhang ZL, Wang Z, Shi XZ, Xu K, Zhang YW, Xu H et al (2015) DAP12 stabilizes the $C$-terminal fragment of the triggering receptor expressed on myeloid cells-2 (TREM2) and protects against LPS-induced pro-inflammatory response. J Biol Chem 290, 15866-15877.

31. Gao X, Dong Y, Liu Z, Niu B (2013) Silencing of triggering receptor expressed on myeloid cells-2 enhances the inflammatory responses of alveolar macrophages to lipopolysaccharide. Mol Med Rep 7, 921-926.

32. Takahashi K, Prinz M, Stagi M, Chechneva O, Neumann H (2007) TREM2-transduced myeloid precursors mediate nervous tissue debris clearance and facilitate recovery in an animal model of multiple sclerosis. Plos Med 4, 675-689.

33. Turnbull IR, Gilfillan S, Cella M, Aoshi T, Miller M, Piccio L, Hernandez M, Colonna M (2006). Cutting edge: TREM-2 attenuates macrophage activation. J Immunol 177, 3520-3524.

34. Henn A, Lund S, Hedtjarn M, Schrattenholz A, Porzgen P, Leist M (2009) The suitability of BV2 cells as alternative model system for primary microglia cultures or for animal experiments examining brain inflammation. Altex Altern Tierexp 26, 83-94. 
35. Ding B, Lin CH, Liu Q, He YY, Ruganzu JB, Jin H, Peng XQ, Ji SF et al (2020) Tanshinone IIA attenuates neuroinflammation via inhibiting RAGE/NF-kappa B signaling pathway in vivo and in vitro. J Neuroinflamm 17, 302.

36. He YY, Ruganzu JB, Lin CH, Ding B, Zheng QZ, Wu XY, Ma RY, Liu Q, Wang Y et al (2020) Tanshinone IIA ameliorates cognitive deficits by inhibiting endoplasmic reticulum stress-induced apoptosis in APP/PS1 transgenic mice. Neurochem Int 133, 104610.

37. Shi Y, Huang W, Wang Y, Zhang R, Hou L, Xu J, Qiu Z, Xie Q et al (2018) Bis(9)-(-)-Meptazinol, a novel dual-binding AChE inhibitor, rescues cognitive deficits and pathological changes in APP/PS1 transgenic mice. Transl Neurodegener 7, 21.

38. Yang WN, Zhang JS, Shi LL, Ji SF, Yang XH, Zhai WY, Zong HF, Qian YH (2019) Protective effects of tanshinone IIA on SH-SY5Y cells against oAbeta1-42-induced apoptosis due to prevention of endoplasmic reticulum stress. Int J Biochem Cell Biol 107, 82-91.

39. Fu AKY, Hung KW, Yuen MYF, Zhou X, Mak DSY, Chan ICW, Cheung TH, Zhang B et al (2016) IL-33 ameliorates Alzheimer's disease-like pathology and cognitive decline. Natl Acad Sci U S A 113, E2705-E2713.

40. Xu Q, Xu W, Cheng H, Yuan H, Tan X et al (2019) Efficacy and mechanism of cGAMP to suppress Alzheimer's disease by elevating TREM2. Brain Behav Immun 81, 495-508.

41. Wirths O, Breyhan H, Marcello A, Cotel MC, Brueck W, Bayer TA (2010) Inflammatory changes are tightly associated with neurodegeneration in the brain and spinal cord of the APP/PS1KI mouse model of Alzheimer's disease. Neurobiol Aging 31, 747-757.

42. Park J, Ha SH, Abekura F, Lim H, Magae J, Ha KT, Chung TW, Chang YC et al (2019) 4-0Carboxymethylascochlorin inhibits expression levels of on inflammation-related cytokines and matrix metalloproteinase-9 through NF-kappa B/MAPK/TLR4 signaling pathway in LPS-activated RAW264.7 cells. Front Pharmacol 10, 1-9.

43. Arendt T (2009) Synaptic degeneration in Alzheimer's disease. Acta Neuropathol 218, 167-179.

44. Giannakopoulos P, Herrmann FR, Bussiere T, Bouras C, Kovari E, Perl DP, Morrison JH, Gold G et al (2003) Tangle and neuron numbers, but not amyloid load, predict cognitive status in Alzheimer's disease. Neurology 60, 1495-1500.

45. Liu Y, Zhang Y, Zheng X, Fang T, Yang X, Luo X, Guo A, Newell KA et al (2018) Galantamine improves cognition, hippocampal inflammation, and synaptic plasticity impairments induced by lipopolysaccharide in mice. J Neuroinflamm 15, 112.

46. Bemiller SM, McCray TJ, Allan K, Formica SV, Xu G, Wilson G, Kokiko Cochran ON, Crish SD et al (2017) TREM2 deficiency exacerbates tau pathology through dysregulated kinase signaling in a mouse model of tauopathy. Mol Neurodegener 12,74.

47. Decourt B, Lahiri DK, Sabbagh MN (2017) Targeting tumor necrosis factor alpha for Alzheimer's disease. Curr Alzheimer Res 14, 412-425. 
48. Koenigsknecht Talboo J, Landreth GE (2005) Microglial phagocytosis induced by fibrillar betaamyloid and IgGs are differentially regulated by proinflammatory cytokines. J Neurosci $25,8240-$ 8249.

49. Lourenco MV, Clarke JR, Frozza RL, Bomfim TR, Forny Germano L, Batista AF, Sathler LB, Brito Moreira $\mathrm{J}$ et al (2013) TNF-alpha mediates PKR-dependent memory impairment and brain IRS-1 inhibition induced by Alzheimer's beta-amyloid oligomers in mice and monkeys. Cell Metab 18, 831843.

50. Clark I, Atwood C, Bowen R, Paz Filho G, Vissel B (2012) Tumor necrosis factor-induced cerebral insulin resistance in Alzheimer's disease links numerous treatment rationales. Pharmacol Rev 64, 1004-1026.

51. Li YK, Liu L, Barger SW, Griffin WST (2003). Interleukin-1 mediates pathological effects of microglia on tau phosphorylation and on synaptophysin synthesis in cortical neurons through a p38-MAPK pathway. J Neurosci 23, 1605-1611.

52. Ringheim GE, Szczepanik AM, Petko W, Burgher KL, Zhu SZ, Chao CC (1998) Enhancement of betaamyloid precursor protein transcription and expression by the soluble interleukin- 6 receptor interleukin-6 complex. Mol Brain Res 55, 35-44.

53. Quintanilla RA, Orellana DI, Gonzalez Billault C, Maccioni RB (2004) Interleukin-6 induces Alzheimertype phosphorylation of tau protein by deregulating the cdk5/p35 pathway. Exp Cell Res 295, 245257.

54. Shankar GM, Li S, Mehta TH, Garcia Munoz A, Shepardson NE, Smith I, Brett FM, Farrell MA et al (2008) Amyloid-beta protein dimers isolated directly from Alzheimer's brains impair synaptic plasticity and memory. Nat Med 14, 837-842.

55. Borchelt DR, Ratovitski T, vanLare J, Lee MK, Gonzales V, Jenkins NA, Copeland NG, Price DL et al (1997). Accelerated amyloid deposition in the brains of transgenic mice coexpressing mutant presenilin 1 and amyloid precursor proteins. Neuron 19, 939-945.

56. Chen CH, Zhou W, Liu S, Deng Y, Cai F, Tone M, Tone Y, Tong Y et al (2012). Increased NF-kappa B signalling up-regulates BACE1 expression and its therapeutic potential in Alzheimer's disease. Int $\mathrm{J}$ Neuropsychopharmacol 15, 77-90.

57. Jay TR, von Saucken VE, Landreth GE (2017) TREM2 in neurodegenerative diseases. Mol Neurodegener 12, 56.

58. Sheng L, Chen M, Cai K, Song Y, Yu D, Zhang H, Xu G (2019) Microglial Trem2 induces synaptic impairment at early stage and prevents amyloidosis at late stage in APP/PS1 mice. Faseb J 33, 10425-10442.

59. Lecca D, Bader M, Tweedie D, Hoffman AF, Jung YJ, Hsueh SC, Hoffer BJ, Becker RE et al (2019) (-)Phenserine and the prevention of pre-programmed cell death and neuroinflammation in mild traumatic brain injury and Alzheimer's disease challenged mice. Neurobiol Dis.130, 104528. 
60. Walter S, Letiembre M, Liu Y, Heine H, Penke B, Hao W, Bode B, Manietta N et al (2007) Role of the toll-like receptor 4 in neuro-inflammation in Alzheimer's disease. Cell Physiol Biochem 20, 947-956.

61. Jeong J, Pandey S, Li Y, Badger JD, Lu W, Roche KW (2019) PSD-95 binding dynamically regulates NLGN1 trafficking and function. Proc Natl Acad Sci U S A 116, 12035-12044.

62. Guarnieri FC, Pozzi D, Raimondi A, Fesce R, Valente MM, Delvecchio VS, Van Esch H, Matteoli M et al (2017) A novel SYN1 missense mutation in non-syndromic X-linked intellectual disability affects synaptic vesicle life cycle, clustering and mobility. Hum Mol Genet 26, 4699-4714.

63. Head E, Corrada MM, Kahle-Wrobleski K, Kim RC, Sarsoza F, Goodus M, Kawas CH (2009) Synaptic proteins, neuropathology and cognitive status in the oldest-old. Neurobiol Aging 30, 1125-1134.

64. Whitfield DR, Vallortigara J, Alghamdi A, Howlett D, Hortobagyi T, Johnson M, Attems J, Newhouse S et al (2014) Assessment of ZnT3 and PSD 95 protein levels in Lewy body dementias and Alzheimer's disease: association with cognitive impairment. Neurobiol Aging 35, 2836-2844.

65. Chugh D, Nilsson P, Afjei SA, Bakochi A, Ekdahl CT (2013) Brain inflammation induces post-synaptic changes during early synapse formation in adult-born hippocampal neurons. Exp Neurol 250, 176188.

66. Rao JS, Kellom M, Kim HW, Rapoport SI, Reese EA (2012) Neuroinflammation and synaptic loss. Neurochem Res 37, 903-910.

67. West MJ, Coleman PD, Flood DG, Troncoso JC (1994) Differences in the pattern of hippocampal neuronal loss in normal ageing and Alzheimer's diseas. Lancet 344, 769-772.

68. Coleman PD, Yao PJ (2003) Synaptic slaughter in Alzheimer's disease. Neurobiol Aging 24, 10231027.

\section{Figures}

\section{Figure 1}

TREM2 is upregulated in APP/PS1 mice. a Scheme of experimental design. $b$ Double immunofluorescence staining of Iba1 and TREM2 in the cortex of 5-month-old APP/PS1 mice. c The mRNA levels of TREM2 in the cortex and hippocampus of 1- to 7-month-old APP/PS1 mice were detected by qRT-PCR. $d$ The protein levels of TREM2 in the cortex and hippocampus of 1- to 7-month-old APP/PS1 mice were assessed by western blotting. $n=10$. Data were presented as mean $\pm S D$ and analyzed by oneway ANOVA followed by Tukey's post-hoc tests. ${ }^{*} p<0.05$ or ${ }^{*} \mathrm{p}<0.01$ vs. 1 -month-old APP/PS1 mice, \#p $<0.05$ or \#\#p $<0.01$ vs. 3-month-old APP/PS1 mice, and $\nabla p<0.01$ vs. 5-month-old APP/PS1 mice. 
Figure 2

a

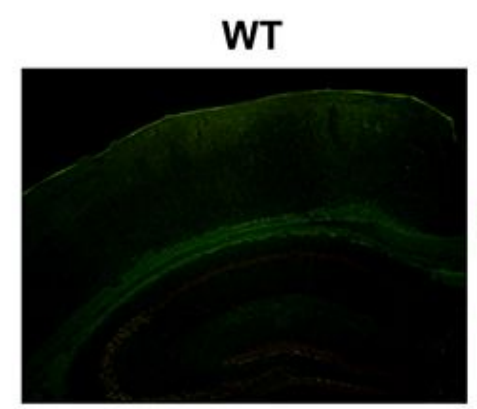

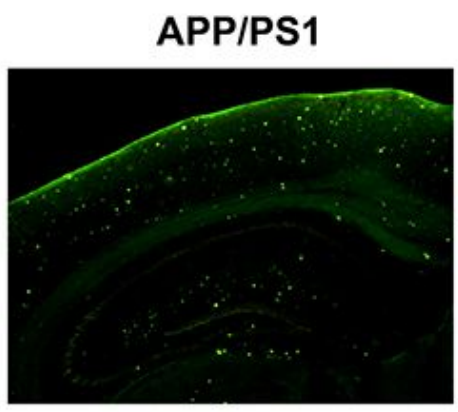

b
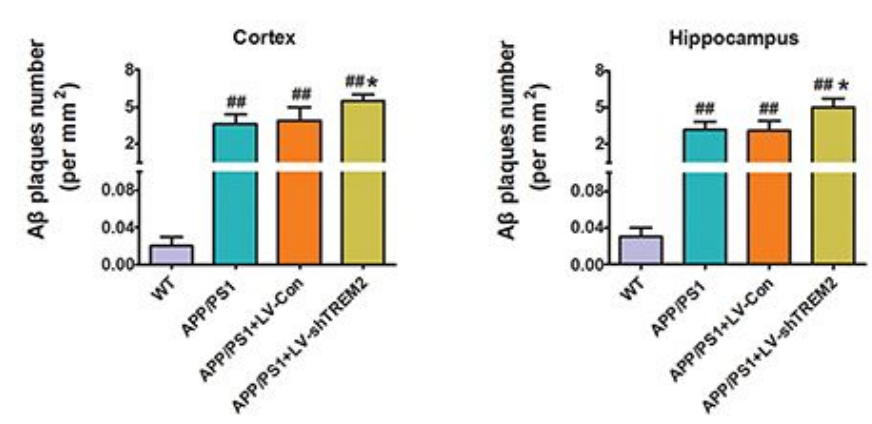

d

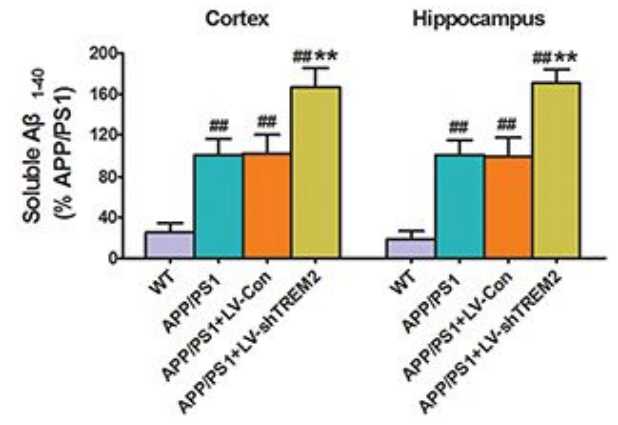

$\mathbf{f}$

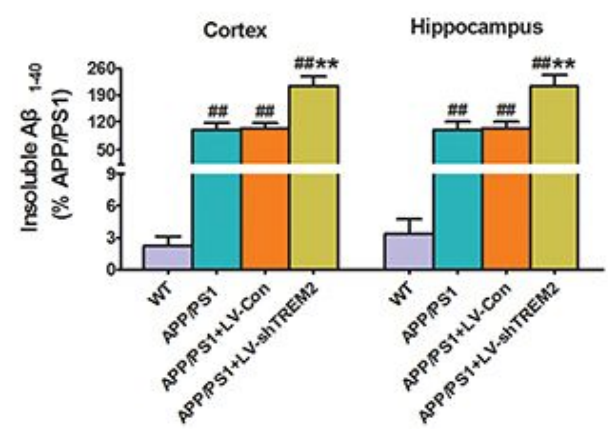

APP/PS1+LV-Con

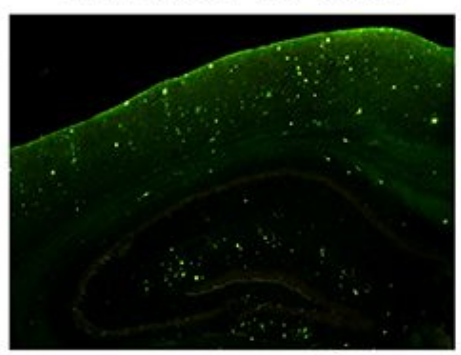

C

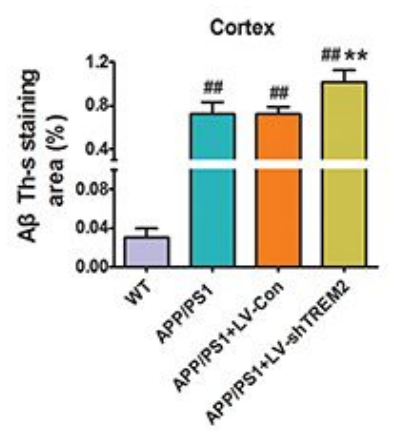

APP/PS1+LV-shTREM2
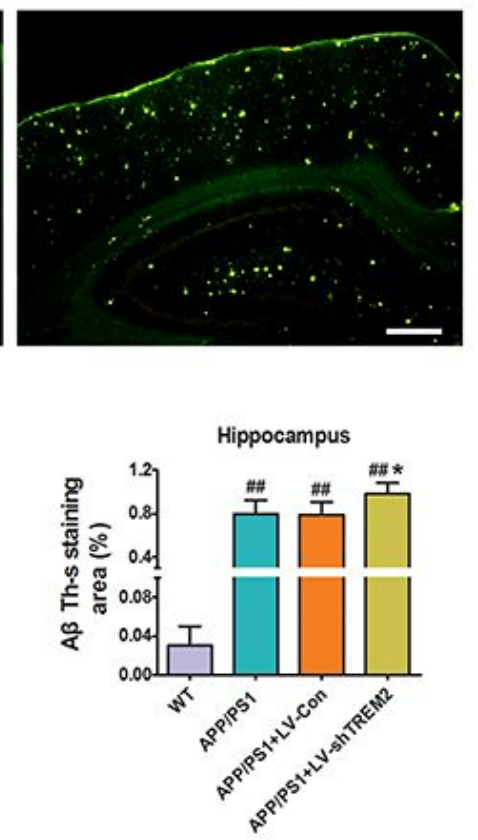

Hippocampus

e

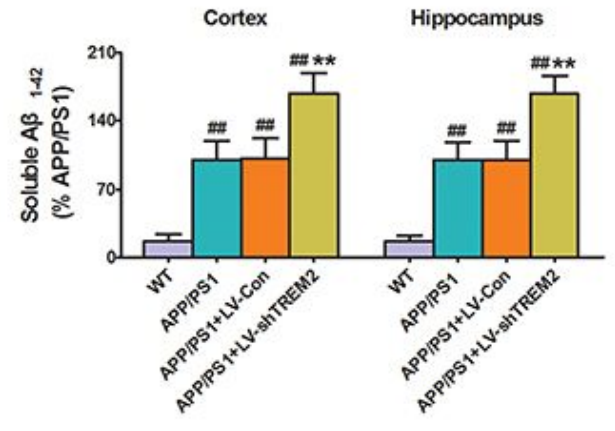

g

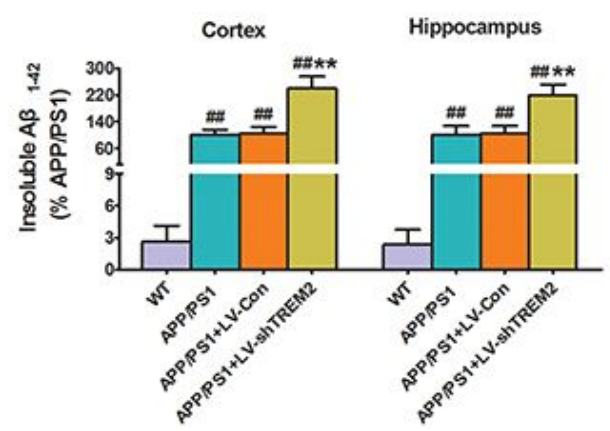

Figure 2

Downregulation of TREM2 expression exacerbated A $\beta$ deposition in APP/PS1 mice. a Representative Th$S$ positive $A \beta$ plaques in the brain. Scale bar $=200 \mu \mathrm{m}$. $b$ The number of $A \beta$ plaques in the cortex and hippocampus. $c$ The $A \beta$ Th-S staining area in the cortex and hippocampus. d-e The protein levels of soluble $A \beta 1-40$ (d) and $A \beta 1-42$ (e) in the cortex and hippocampus. $f-g$ The protein levels of insoluble $A \beta 1-$ $40(\mathrm{f})$ and $A \beta 1-42(\mathrm{~g})$ in the cortex and hippocampus. $n=5$. Data were presented as mean \pm SD and 
analyzed by one-way ANOVA followed by Tukey's post-hoc tests. \#\#p $<0.01$ vs. WT group, and *p $<0.05$ or **p $<0.01$ vs. APP/PS1 group.

Figure 3

a

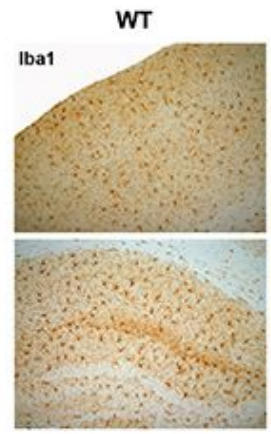

b

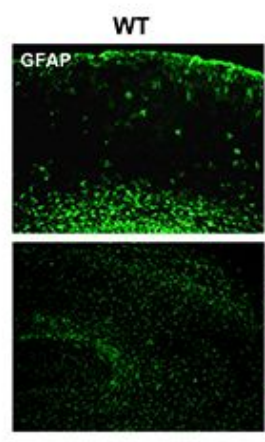

APP/PS1

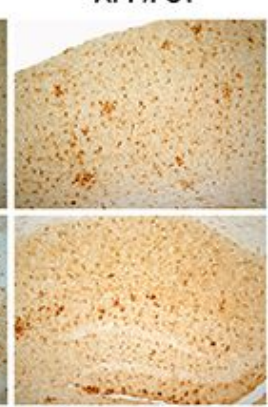

APPIPS1

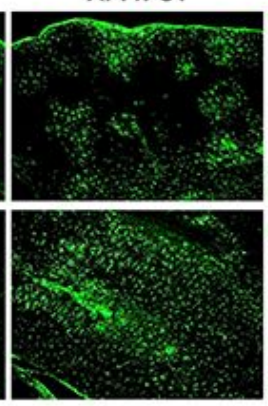

APPIPS1+LV-Con
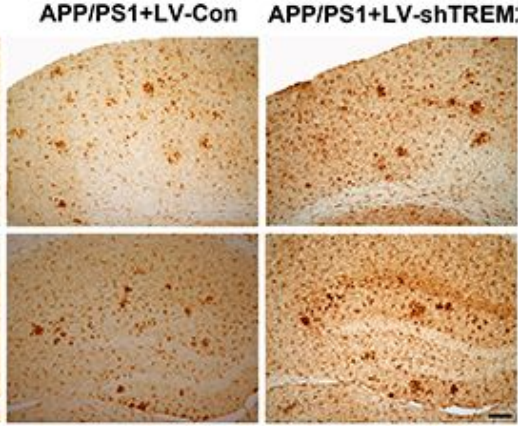

APPIPS1+LV-Con APPIPS1+LV-ShTREM2

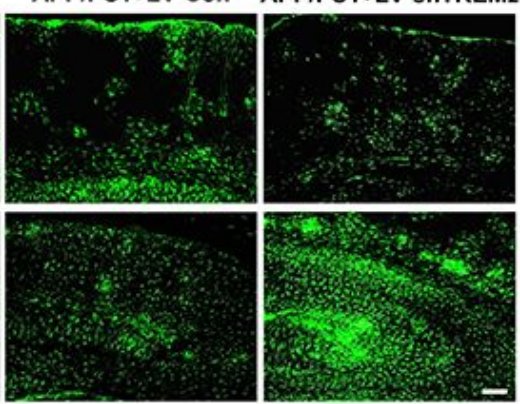

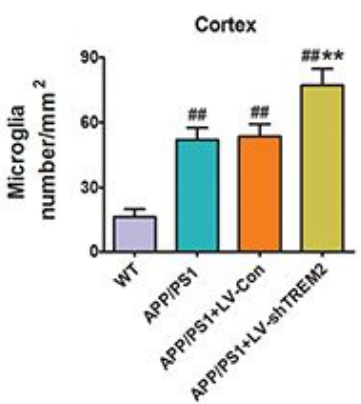

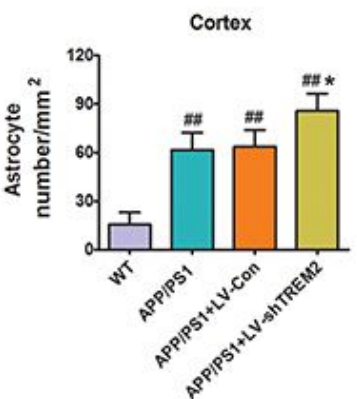

d
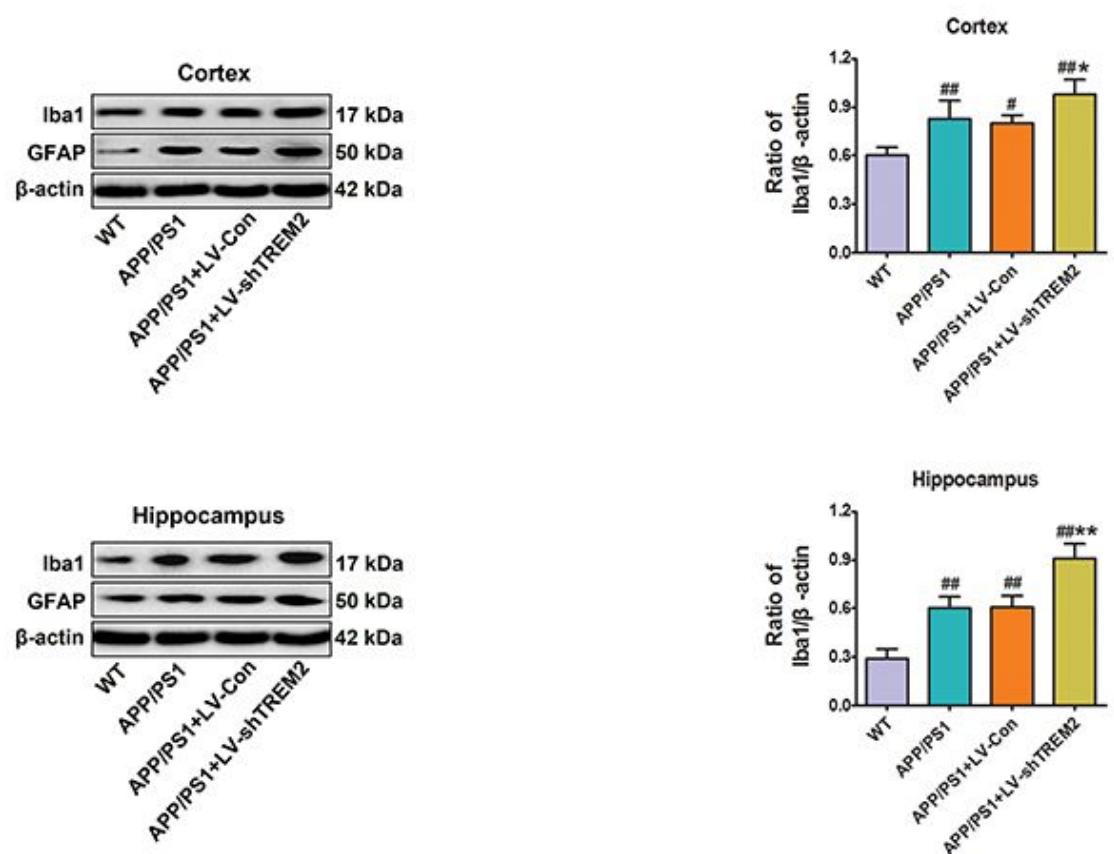
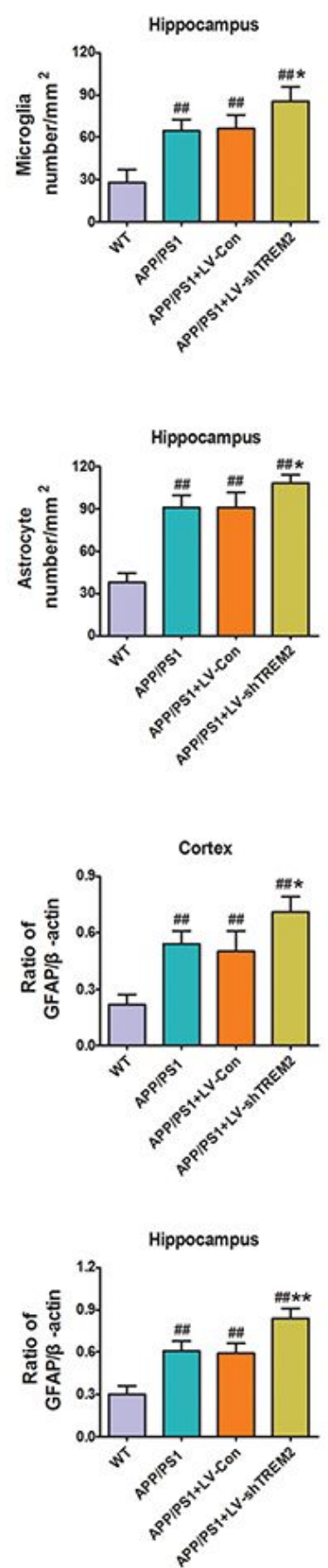

\section{Figure 3}

Downregulation of TREM2 expression induced microgliosis and astrogliosis in APP/PS1 mice. a Representative immunohistochemistry staining of Iba1 and quantitative analysis of microglia in the cortex and hippocampus. Scale bar $=200 \mu \mathrm{m}$. b Representative immunofluorescence staining of GFAP and quantitative analysis of astrocytes in the cortex and hippocampus. Scale bar $=200 \mu \mathrm{m}$. c Western 
blotting and densitometry analysis of Iba1and GFAP in the cortex. $\mathrm{d}$ Western blotting and densitometry analysis of Iba1and GFAP in the hippocampus. $n=5$. Data were presented as mean $\pm S D$ and analyzed by one-way ANOVA followed by Tukey's post-hoc tests. \#p $<0.05$ or \#\#p $<0.01$ vs. WT group, and * $p<$ 0.05 or ${ }^{*} p<0.01$ vs. APP/PS1 group.

\section{Figure 4}

Cortex

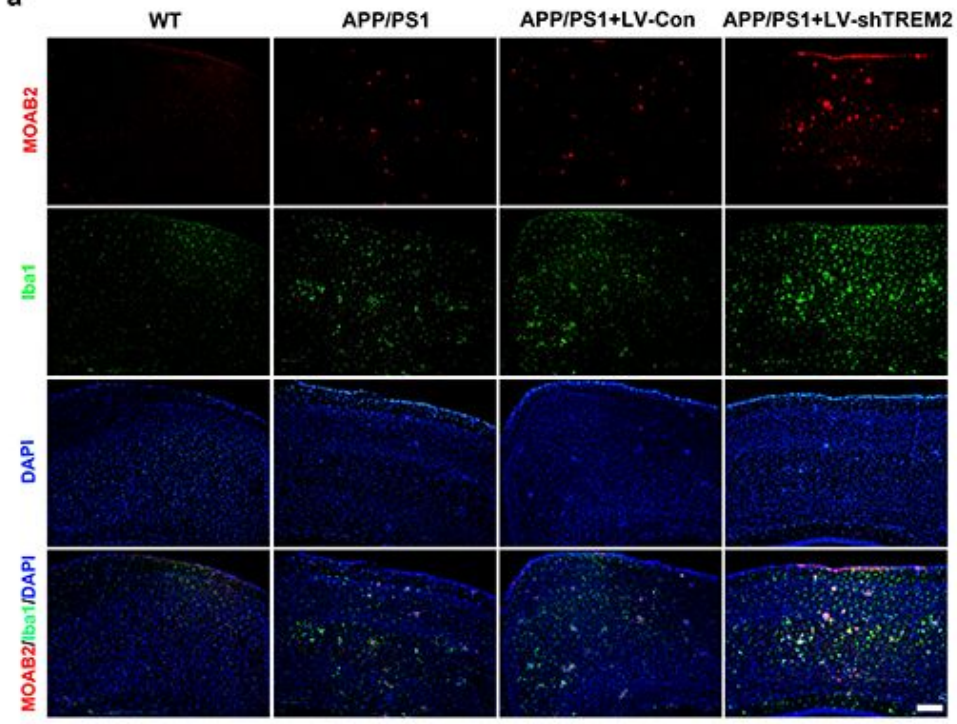

Hippocampus

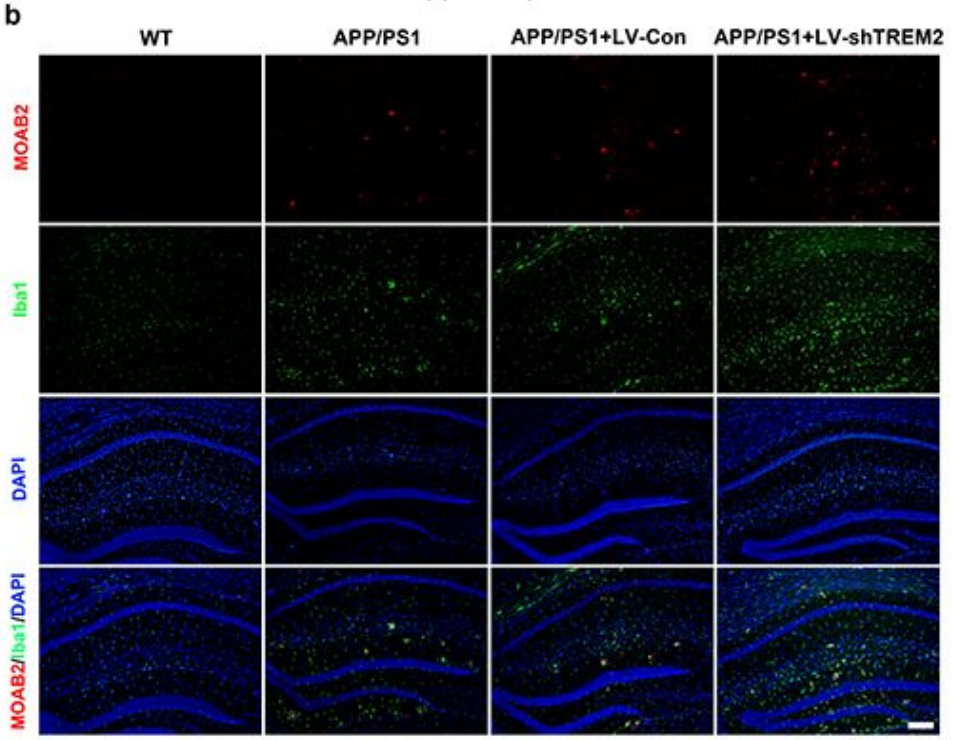

\section{Figure 4}

Reactive microglia surround A $\beta$ plaques in APP/PS1 mice. a Immunofluorescence staining of MOAB2positive $A \beta$ (red), Iba1 (green), and DAPI (blue) in the cortex. b Immunofluorescence staining of MOAB2positive AB (red), Iba1 (green), and DAPI (blue) in the hippocampus. Scale bar $=200 \mu \mathrm{m} . \mathrm{n}=5$.

Figure 5

a

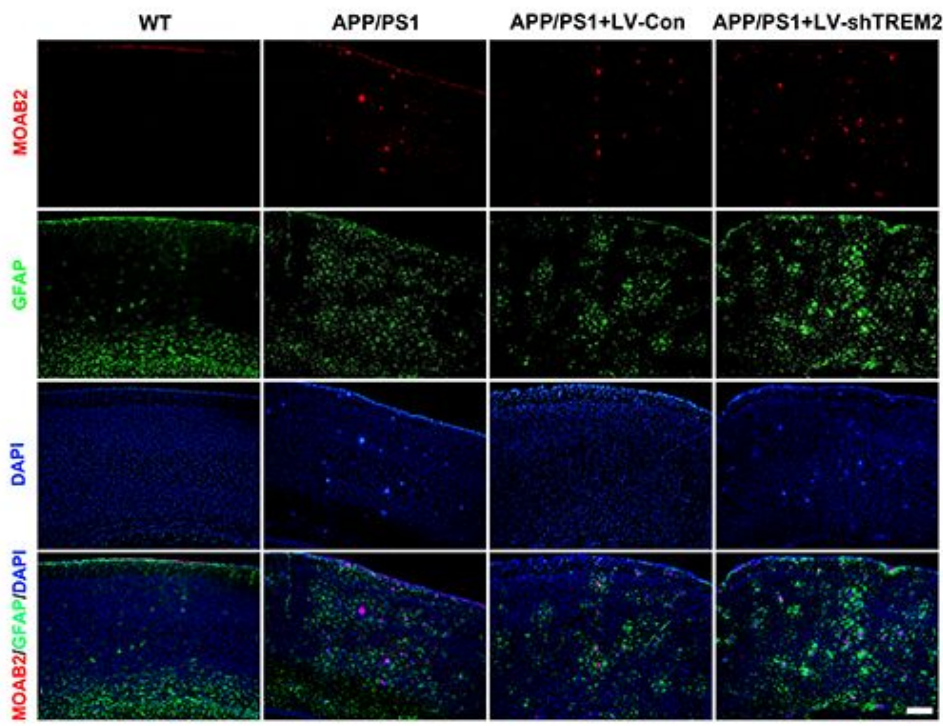

b

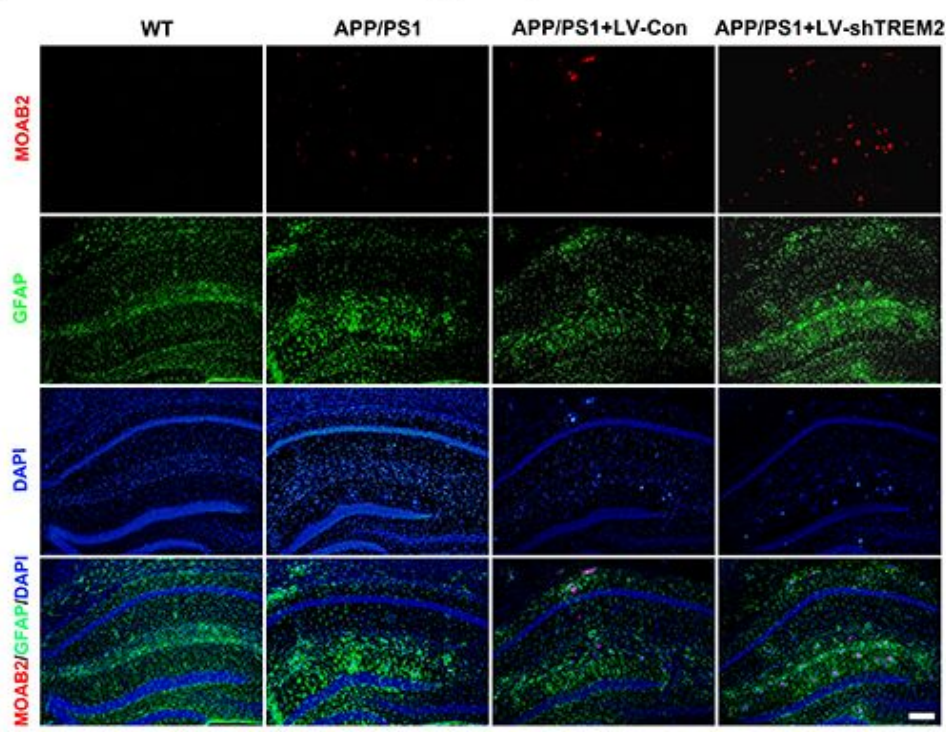

Figure 5 
Reactive astrocytes surround A $\beta$ plaques in APP/PS1 mice. a Immunofluorescence staining of MOAB2positive $A \beta$ (red), GFAP (green), and DAPI (blue) in the cortex. b Immunofluorescence staining of MOAB2positive A $($ red), GFAP (green), and DAPI (blue) in the hippocampus. Scale bar $=200 \mu \mathrm{m} . n=5$.

Figure 6

a

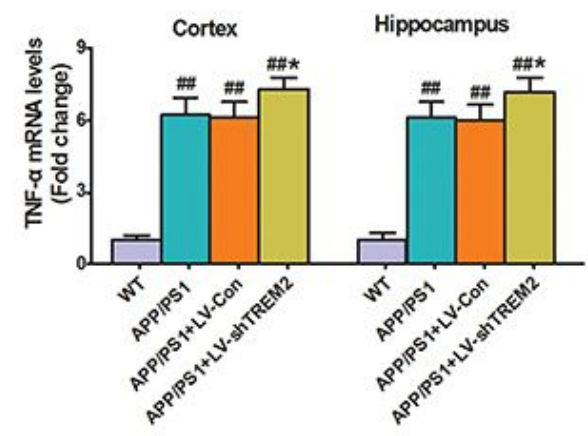

b

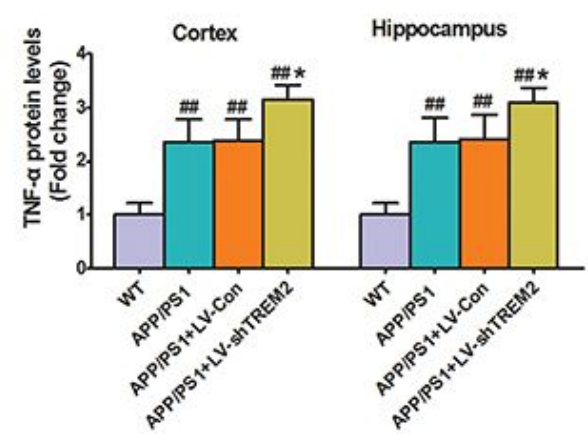

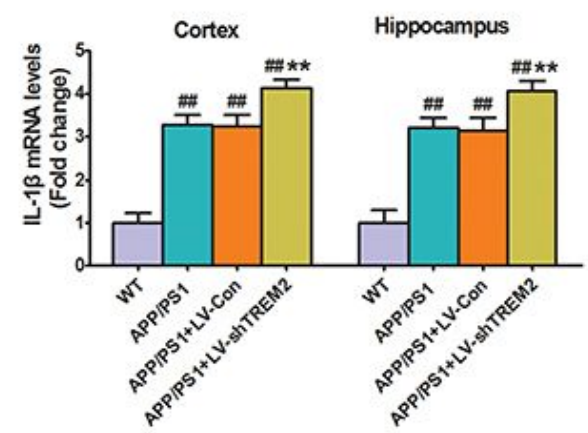

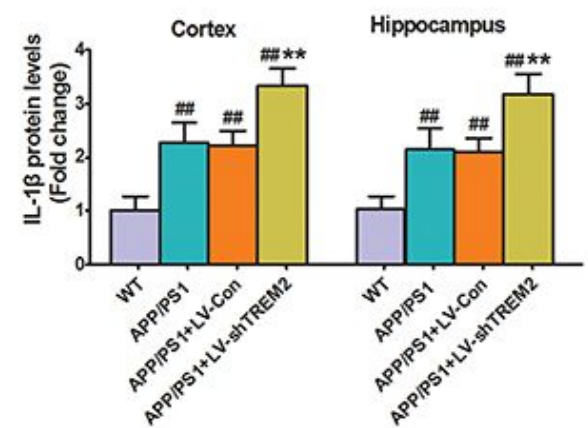

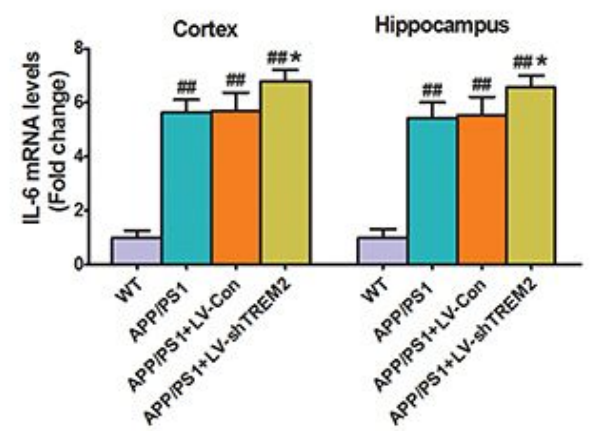

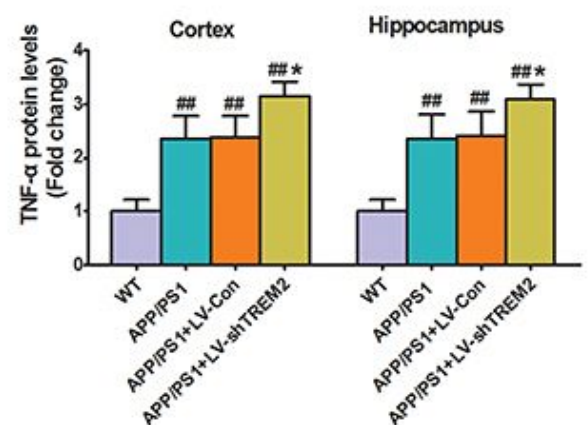

Figure 6

Downregulation of TREM2 expression promoted pro-inflammatory cytokines production in APP/PS1 mice. a The mRNA levels of TNF- $a, \mathrm{IL}-1 \beta$, and IL- 6 in the cortex and hippocampus were detected by qRTPCR. $b$ The protein levels of TNF-a, IL-1 $\beta$, and IL- 6 in the cortex and hippocampus were measured by ELISA. $n=5$. Data were presented as mean $\pm S D$ and analyzed by one-way ANOVA followed by Tukey's post-hoc tests. \#\#p $<0.01$ vs. WT group, and $\star_{p}<0.05$ or $* * p<0.01$ vs. APP/PS1 group.

\section{Figure 7}

Downregulation of TREM2 expression induced TLR4-mediated MAPK signaling pathway activation in APP/PS1 mice. a-b Western blotting and densitometry analysis of TLR4, MyD88, and TRAF6 in the cortex (a) and hippocampus (b). c-d Western blotting and densitometry analysis of phospho-p38, total-p38, phospho-JNK, total-JNK, phospho-ERK1/2, and total-ERK1/2 in the cortex (c) and hippocampus (d). $\beta$ actin was used as a loading control. $n=5$. Data were presented as mean $\pm S D$ and analyzed by one-way ANOVA followed by Tukey's post-hoc tests. \#p $<0.05$ or \#\#p $<0.01$ vs. WT group, and * $p<0.05$ or ** $p<$ 0.01 vs. APP/PS1 group. 
Figure 8

a
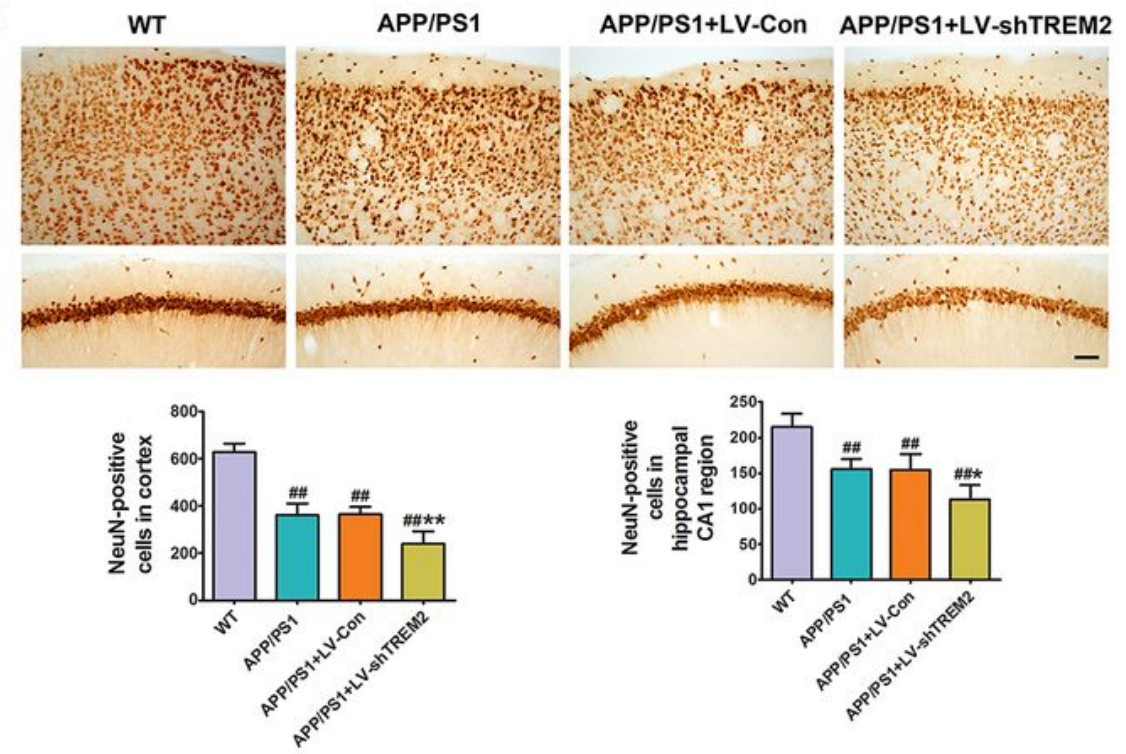

b
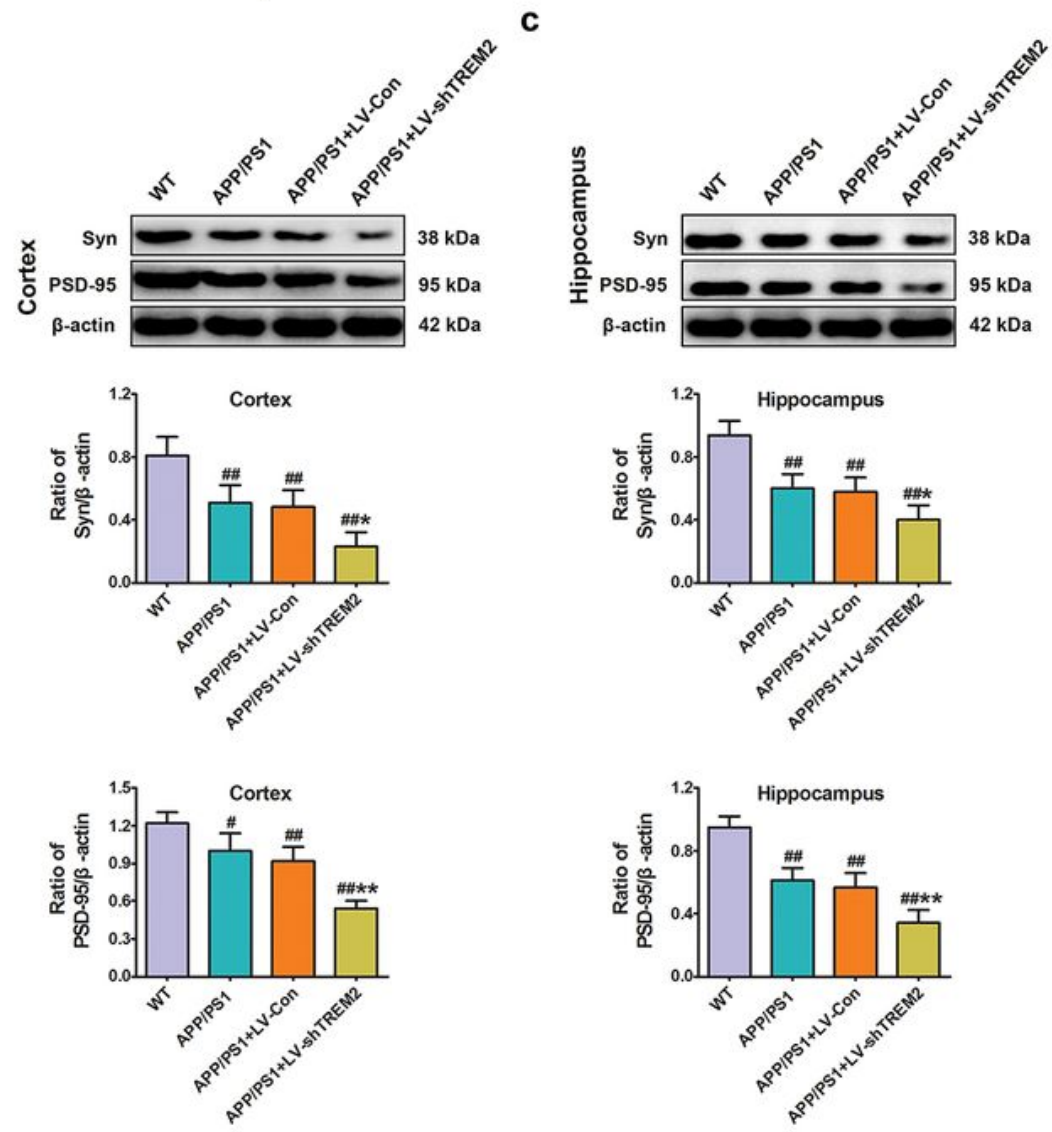

\section{Figure 8}

Downregulation of TREM2 expression promoted neuronal and synaptic loss in APP/PS1 mice. a Representative immunohistochemistry staining of NeuN and quantitative analysis of neurons in the cortex and hippocampal CA1 region. Scale bar $=200 \mu \mathrm{m}$. b-c Western blotting and densitometry analysis of Syn and PSD-95 in the cortex (b) and hippocampus (c). $\beta$-actin was used as a loading control. $n=5$. 
Data were presented as mean \pm SD and analyzed by one-way ANOVA followed by Tukey's post-hoc tests. $\# p<0.05$ or $\# \# p<0.01$ vs. WT group, and * $p<0.05$ or ** $p<0.01$ vs. APP/PS1 group.

\section{Figure 9}

a

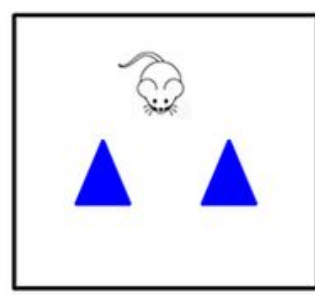

Acquisition

b

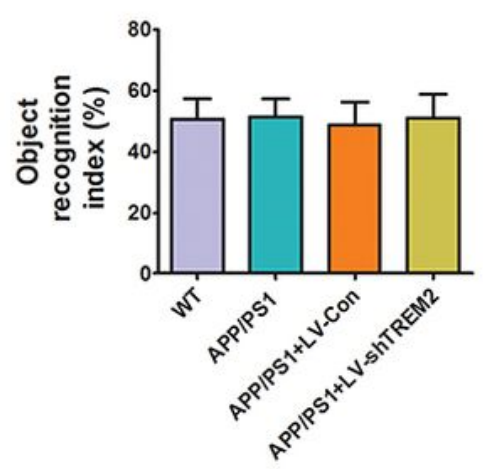

e

$\mathbf{f}$
C

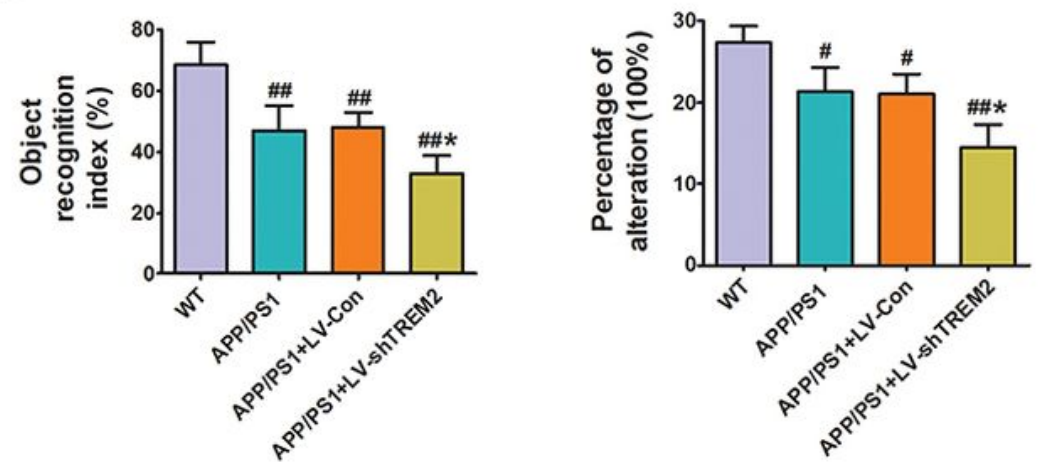

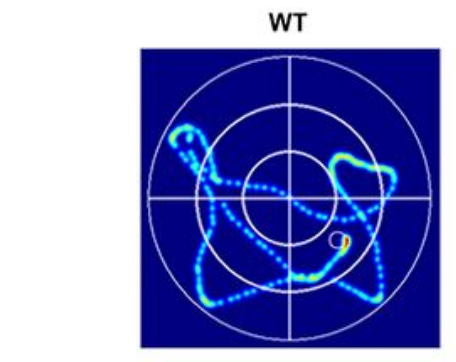

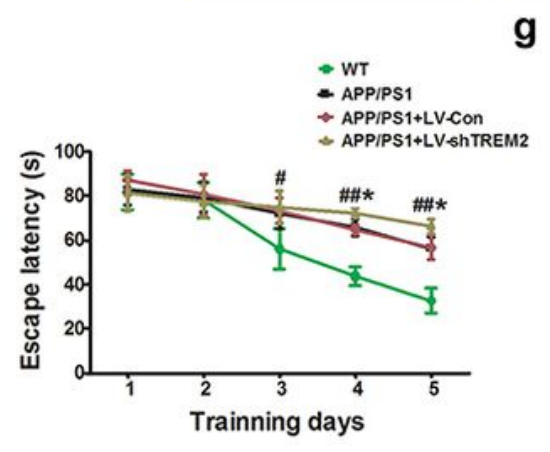

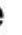

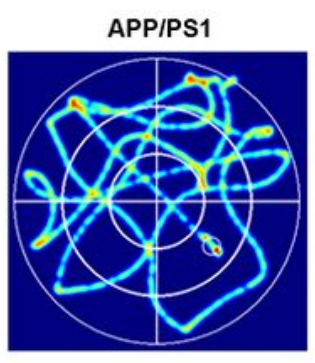

g

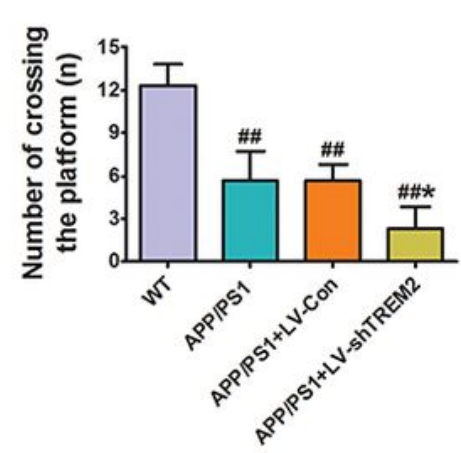

d

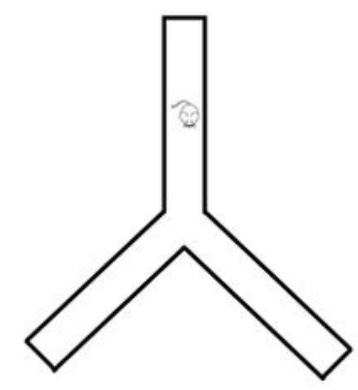

Testing

APP/PS1+LV-Con

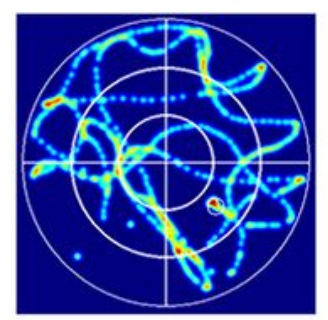

APP/PS1+LV-shTREM2

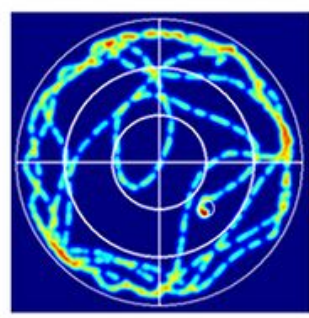

h

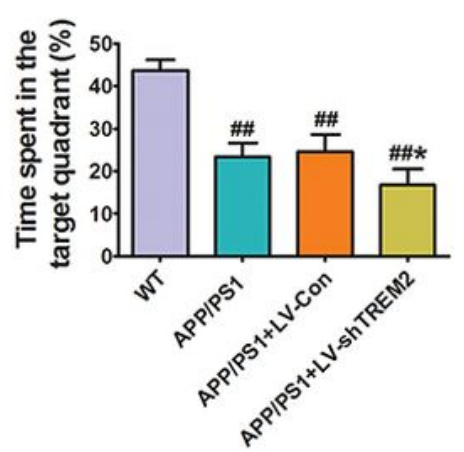

Figure 9

Downregulation of TREM2 expression exacerbated cognitive deficits in APP/PS1 mice. a Representative diagram of NOR test. b-c Recognition index of mice during acquisition phase (b) and testing phase (c). d The percentage of spontaneous alternation in Y-maze analysis. e-h Morris water maze test. 
Representative path tracings of each group in the hidden platform test (e), escape latency (f), number of crossing the platform $(\mathrm{g})$, and the percentage of time spent in the target quadrant $(h) . n=10$. Differences in the escape latency were analyzed using a two-way repeated-measures ANOVA followed by Tukey's post-hoc tests. The other data were analyzed by one-way ANOVA followed by Tukey's post-hoc tests. \#p< 0.05 or $\# \# p<0.01$ vs. WT group, and * $p<0.05$ vs. APP/PS1 group.

\section{Figure 10}

a

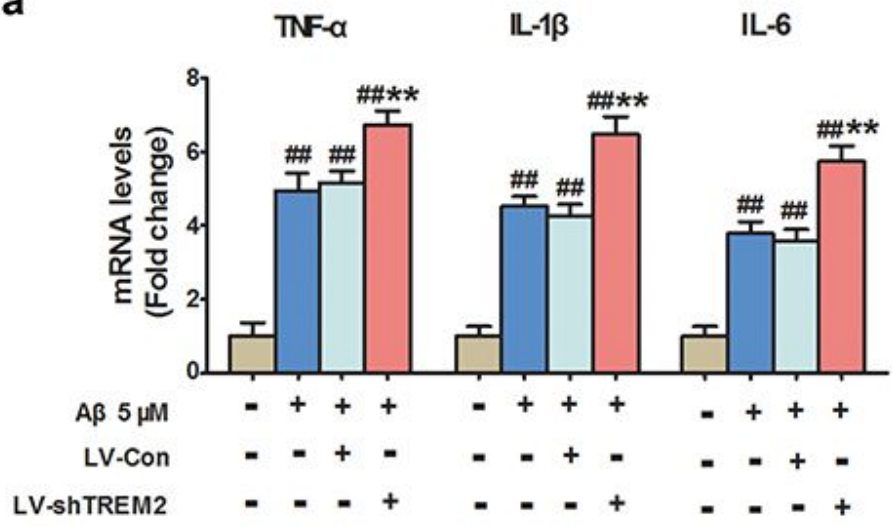

b

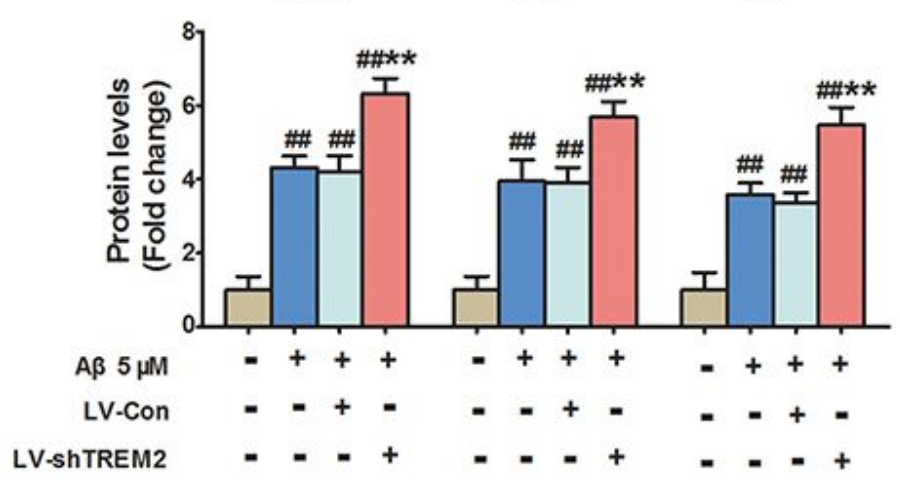

Figure 10

Knockdown of TREM2 expression increased pro-inflammatory cytokines production in A 1 1-42-induced BV2 cells. a The mRNA levels of TNF-a, IL-1 $\beta$, and IL- 6 in BV2 cells were detected by qRT-PCR. $b$ The protein levels of TNF-a, IL-1 $\beta$, and IL- 6 in culture medium from BV2 cells were detected by ELISA. $n=5$. Data were presented as mean \pm SD and analyzed by one-way ANOVA followed by Tukey's post-hoc tests. $\# \# p<0.01$ vs. control, and ${ }^{*} p<0.01$ vs. $A \beta 1-42$ treated cells.

Figure 11
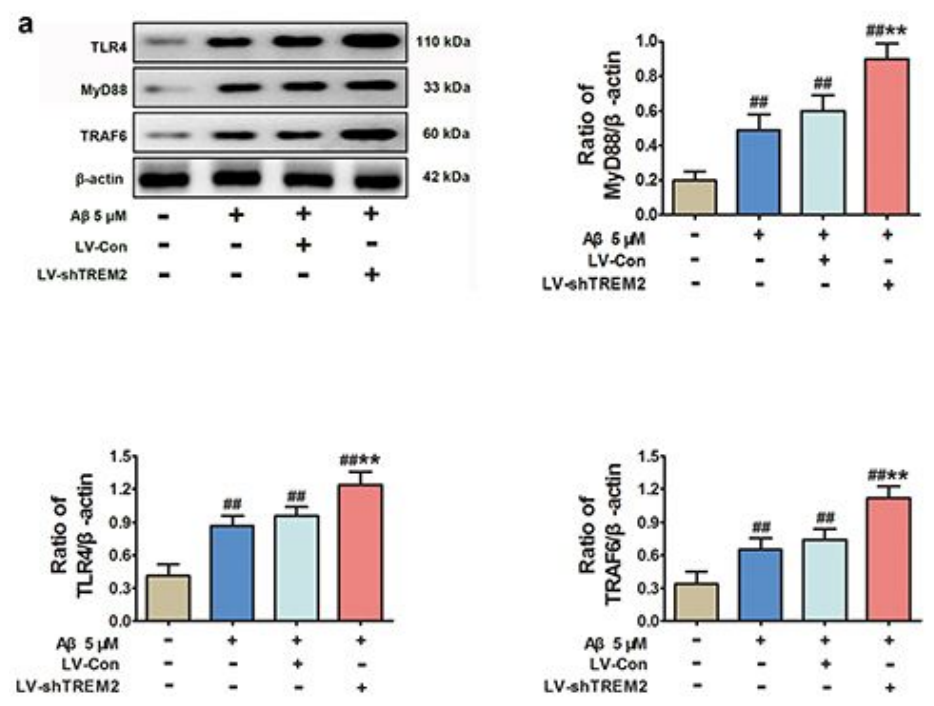
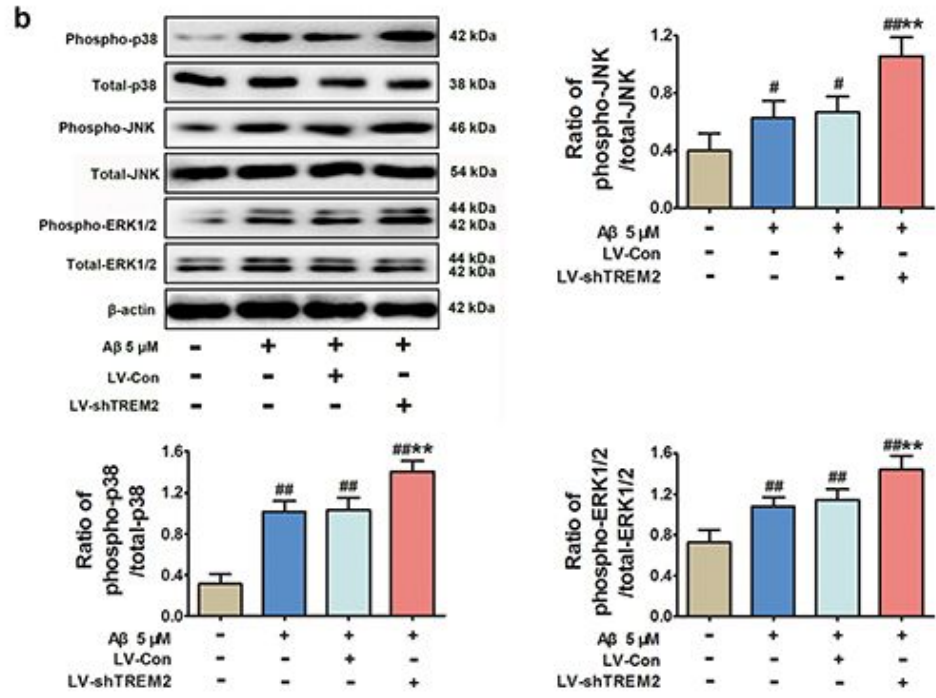

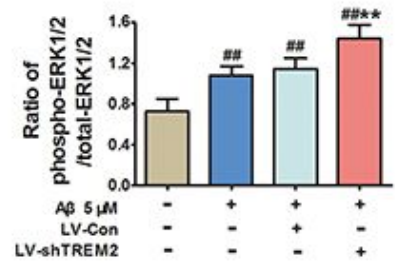




\section{Figure 11}

Knockdown of TREM2 expression aggravated A 1 1-42-induced inflammatory responses via TLR4mediated MAPK signaling pathway in BV2 cells. a Western blotting and densitometry analysis of TLR4, MyD88, and TRAF6. b Western blotting and densitometry analysis of phospho-p38, total-p38, phosphoJNK, total-JNK, phospho-ERK1/2, and total-ERK1/2. $\beta$-actin was used as a loading control. $n=5$. Data were presented as mean \pm SD and analyzed by one-way ANOVA followed by Tukey's post-hoc tests. \#p < 0.05 or \#\#p $<0.01$ vs. control, and ${ }^{* *} p<0.01$ vs. $A \beta 1-42$ treated cells.

Figure 12

a

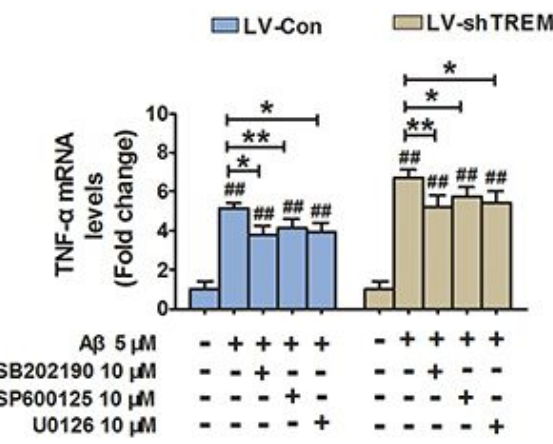

b

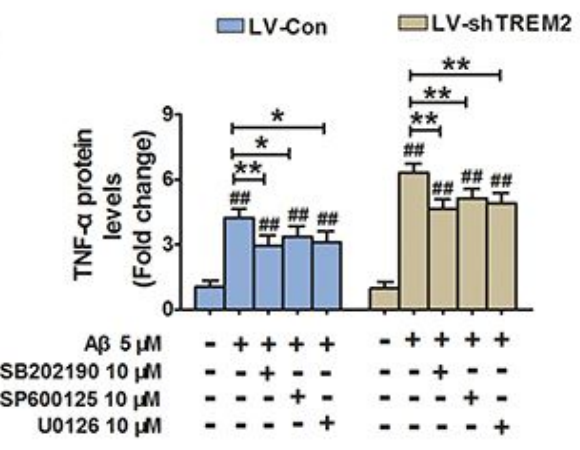

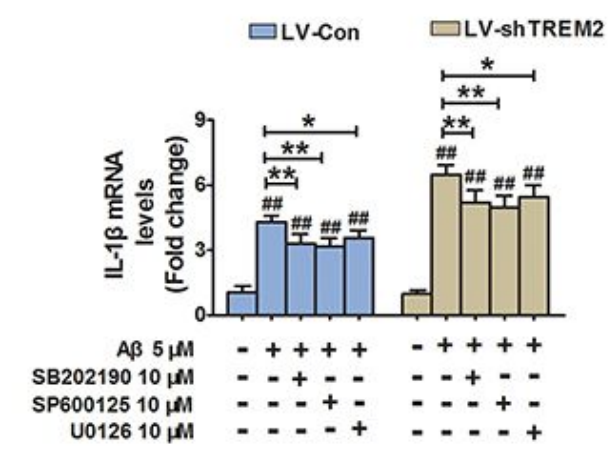
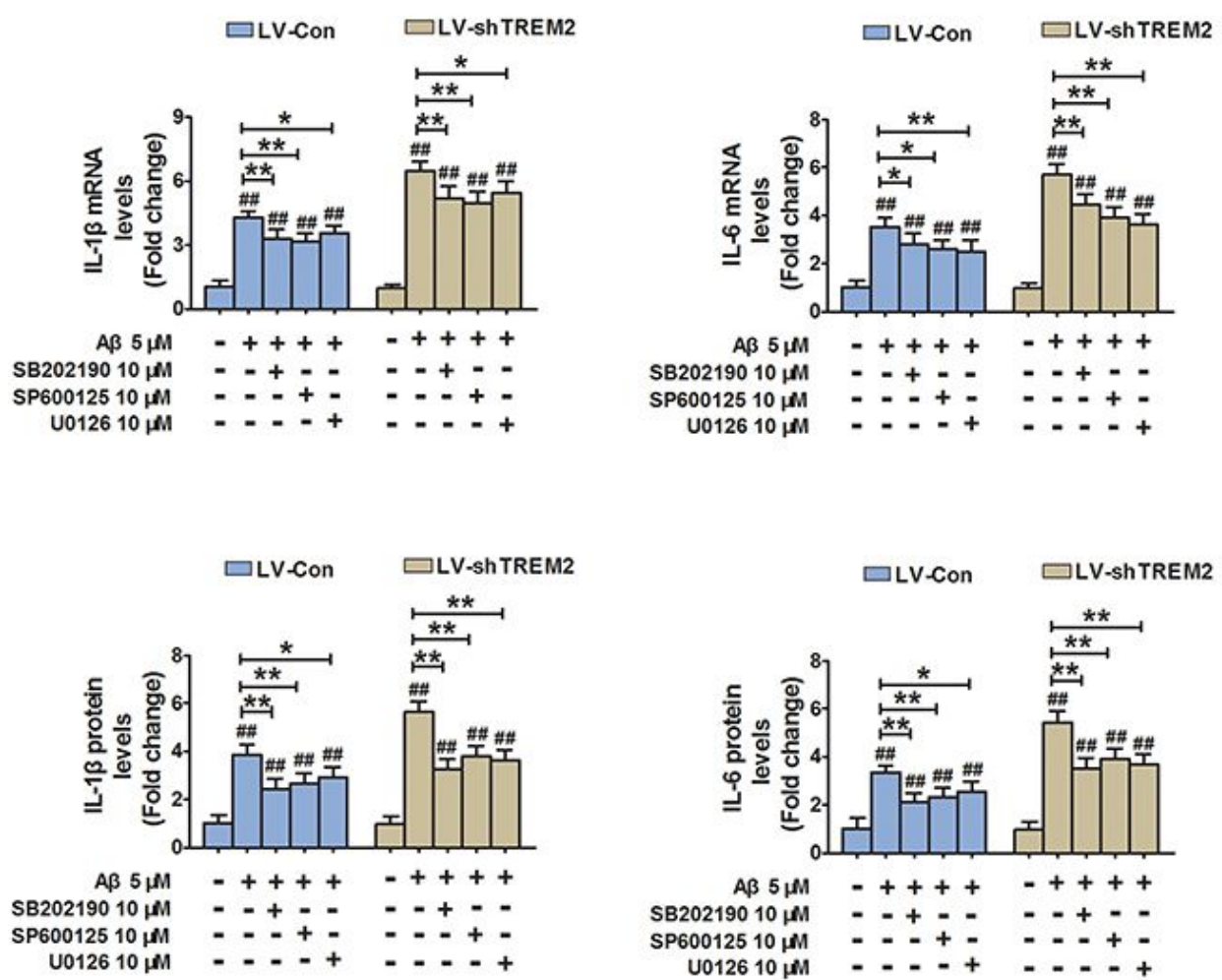

Figure 12

p38, JNK, and ERK1/2 inhibitors suppressed A 1 1-42-induced pro-inflammatory cytokines production in TREM2-knocked down BV2 cells. a The mRNA levels of TNF- $a, \mathrm{IL}-1 \beta$, and IL- 6 in BV2 cells were detected by qRT-PCR. $b$ The protein levels of TNF- $a$, IL-1 $\beta$, and IL- 6 in culture medium from BV2 cells were measured by ELISA. $n=5$. Data were presented as mean $\pm S D$ and analyzed by one-way ANOVA followed by Tukey's post-hoc tests. \#\#p $<0.01$ vs. control, and ${ }^{*} p<0.05$ or ${ }^{\star \star} p<0.01$ vs. A $\beta 1-42$ treated cells.

\section{Supplementary Files}

This is a list of supplementary files associated with this preprint. Click to download.

- SupplementaryFigurecaptions.docx 
- SupplementaryFig.1.tif

- SupplementaryFig.2.tif 